\title{
REPRESENTATIONS OF COMPLEMENTED MODULAR LATTICES
}

\author{
BY \\ BJARNI JONSSON(1)
}

Introduction. It was proved by von Neuman [10, vol. II] that a ring $R$ is regular if and only if the set $L(R)$ of all principal left ideals of $R$ is a complemented modular lattice under the usual operations of addition and intersection of ideals. We shall here be concerned with the problem, also investigated by von Neumann, of finding for a given complemented modular lattice $B$ a regular ring $R$ such that $B \cong L(R)$. As von Neumann observed, this may be regarded as a generalization of the problem of introducing coordinates in a projective geometry, and we shall therefore say that $B$ is COORDINATIZABLE if and only if such a ring $R$ exists.

A complete solution to our problem would consist in an axiomatic characterization of the class $\boldsymbol{K}$ of all coordinatizable lattices. However, this seems to be an extremely difficult problem, and in fact it is doubtful that any reasonable axiom system can be found. In any case, as we observed in [4], no finite set of first order sentences could possibly serve as an axiom system for $\boldsymbol{K}$. We shall therefore be concerned with the less ambitious problem of trying to find sufficient conditions in order for a lattice $B$ to be coordinatizable. Our principal result will be a generalization of von Neumann's theorem which asserts that every complemented modular lattice which has a homogeneous $n$-frame with $n \geqq 4$ is coordinatizable. In particular, it follows from our results that, with certain well known exceptions in the lowest dimensions, every simple complemented modular lattice is coordinatizable.

Generalizing von Neumann's concept of a normal $n$-frame of a complemented modular lattice, we shall introduce the notions of a PARTIAL $n$-FRAME and a LARGE PARTIAL $n$-FRAME, and our principal result states that every complemented Arguesian lattice $B$ possessing a large partial three-frame is coordinatizable. Essential use will be made of Frink's representation (cf. [3]) of a complemented modular lattice as a sublattice of the lattice of all subspaces of a projective geometry, and of the resulting representation (cf. [5]) of a complemented Arguesian lattice as a sublattice of the lattice of all subgroups of an Abelian group. This latter representation is used to prove that every complemented Arguesian lattice which possesses a large partial three-

Received by the editors September 19, 1959.

(1) These investigations were supported in part by a grant from the National Science Foundation. The principal results contained in this paper were announced at the Symposium on Lattice Theory in Monterey, California, April 16-17, 1959 and they appear, without proofs, in [4]. 
frame can be embedded as an ideal in a complemented Arguesian lattice with a normal $n$-frame where $n \geqq 4$. The proof of this embedding theorem is quite involved, but once it has been established our principal result follows immediately from von Neumann's theorem together with the fact that every principal ideal of a coordinatizable lattice is coordinatizable.

All the lattices considered here will be assumed to have a zero element 0 , which will be regarded as a distinguished element. That is, when speaking of a sublattice of a given lattice we mean a sublattice containing the zero element of the original lattice, and when speaking of a homomorphism of one lattice into another we shall assume that the zero element of the first lattice is mapped onto the zero element of the second. We shall use + and - for the binary operations of lattice addition and multiplication, $\sum$ and $\prod$ for the corresponding operations on finite sequences, and $\leqq$ for the inclusion relation. If $b \leqq a$, then $a / b$ is the lattice quotient consisting of all elements $x$ with $b \leqq x \leqq a$. In particular $a / 0$ is the principal ideal generated by $a$, but there will be occasions when this last notation is ambiguous because our arguments will concern two lattices $A$ and $B$, where $B$ is a sublattice of $A$, and an element $a \in B$. We shall therefore use complex multiplication and write $B a$ and $A a$ for the principal ideals of $B$ and of $A$ which are generated by $a$.

If the elements $a_{0}, a_{1}, \cdots, a_{n-1}$ of a modular lattice $B$ are independent we write $\left(a_{0}, a_{1}, \cdots, a_{n-1}\right) \perp$. If the terms of a sum are independent, we also indicate this by writing $\dot{+}$ and $\dot{\Sigma}$ in place of + and $\sum$. Thus

$$
\begin{aligned}
& a \dot{+} b=a+b, \\
& \sum_{i<n} a_{i}=a_{0} \dot{+} a_{1} \dot{+} \cdot+a_{n-1}=\sum_{i<n} a_{i},
\end{aligned}
$$

in case $a b=0$ and $\left(a_{0}, a_{1}, \cdots, a_{n-1}\right) \perp$. The relations of perspectivity and of projectivity, between quotients or between elements, will be denoted by $\sim$ and $\approx$, and we shall write $a<b$ in case $a$ is perspective to a part of $b$, i.e., $a \sim x$ for some $x \leqq b$. As before, there may be ambiguity when two different lattices are being considered, and we shall then write $a \sim b$ in $B$ in place of $a \sim b$, and similarly for the other two relations.

1. Partial $n$-frames. Although we are primarily interested in complemented modular lattices, it will be important to introduce the notion of a partial $n$-frame for an arbitrary modular lattice with a unit element.

Definition 1.1. Suppose $B$ is a modular lattice with a unit element $u$, and $n$ is a positive integer. By a PARTIAL $n$-FRAME of $B$ we mean an ordered triple $a=\langle b, c, d\rangle$ where $b$ is an $n$-termed sequence whose terms $b_{i}, i=0,1, \cdots$, $n-1$, belong to $B, c$ is a symmetric $n \times n$ matrix whose terms $c_{i, j}, i, j$ $=0,1, \cdots, n-1$, belong to $B, d$ is an element of $B$, and the following conditions are satisfied: 
(i) $u=\dot{\sum}_{i<n} b_{i} \dot{+} d$.

(ii) $b_{i}+b_{j}=b_{i}+c_{i, j}$ for $i, j=0,1, \cdots, n-1$.

(iii) $c_{i, j}=\left(c_{i, p}+c_{p, j}\right)\left(b_{i}+b_{j}\right)$ for $i, j, p=0,1, \cdots, n-1$.

If $a=\langle b, c, d\rangle$ is a partial $n$-frame of $B$, we agree to write

$$
a_{i}=b_{i}, \quad a_{i, j}=c_{i, j}, \quad a^{*}=d, \quad \bar{a}_{i}=\sum_{i \neq j<n} a_{j}+a^{*} .
$$

A partial $n$-frame $a$ of $B$ is said to be LARGE in case $a^{*}$ is the sum of finitely many elements, each of which is perspective to a part of $a_{0}$.

By a GLOBAL $n$-FRAME of $B$ we mean a partial $n$-frame $a$ of $B$ such that $a^{*}=0$.

Clearly, if $a$ is a partial $n$-frame of $B$, then $a_{i, i}=0, a_{i}$ and $a_{j}$ are perspective with $a_{i, j}$ as axis of perspectivity, $u=a_{i} \dot{+} \bar{a}_{i}=a_{i, j}+\bar{a}_{i}$ provided $i \neq j$, and

$$
\bar{a}_{i} \bar{a}_{j}=\sum_{i, j \neq p<n} a_{p}+a^{*} .
$$

Furthermore, although $B$ is not assumed to be complemented, several elementary facts concerning $n$-frames generalize to the present situation. As an example, if

$$
u=\sum_{i<n} b_{i} \dot{+} d,
$$

and if $b_{0}+b_{i}=b_{0}+x_{i}=b_{i}+x_{i}$ for $i=1,2, \cdots, n-1$, then there exists a unique partial $n$-frame $a$ of $B$ such that $a_{i}=b_{i}$ for $i=0,1, \cdots, n-1, a_{0, i}=x_{i}$ for $i=1,2, \cdots, n-1$, and $a^{*}=d$.

LeMma 1.2. For $n \geqq 3$, if $a$ is a partial $n$-frame of a complemented modular lattice $B$, then the set $B \bar{a}_{0} \cup\left\{a_{0}, a_{0,1}\right\}$ generates $B$.

Proof. Let $C$ be the lattice generated by $B \bar{a}_{0} \cup\left\{a_{0}, a_{0,1}\right\}$. Since $a_{0}$ and $\bar{a}_{0} \bar{a}_{1}$ are elements of $C$, the element $\bar{a}_{1}=a_{0}+\bar{a}_{0} \bar{a}_{1}$ also belongs to $C$. The perspectivity

$$
x \rightarrow x^{\prime}=\bar{a}_{1}\left(x+a_{0,1}\right)
$$

maps $B \bar{a}_{0}$ isomorphically onto $B \bar{a}_{1}$, and since $x^{\prime} \in C$ whenever $x \in C$, it follows that $B \bar{a}_{1} \subseteq C$. The element $a_{0,2}$ belongs to $C$, and replacing the subscript 1 by 2 in the above argument we infer that $B \bar{a}_{2} \subseteq C$.

Assuming that $x \in B$ and $x \bar{a}_{0}=0$, let

$$
y=\bar{a}_{1}\left(x+a_{1}\right), \quad z=\bar{a}_{2}\left(x+a_{2}\right) .
$$

Then $y \in B \bar{a}_{1}$ and $z \in B \bar{a}_{2}$, hence $y, z \in C$. Furthermore

$$
\left(a_{1}+y\right)\left(a_{2}+z\right)=\left(x+a_{1}\right)\left(x+a_{2}\right)=x
$$


because $\left(a_{1}, a_{2}, x\right) \perp$. Consequently $x \in C$. Finally, for any $b \in B$ there exists $x \in B$ such that $b=b \bar{a}_{0}+x$. Then $b \bar{a}_{0} \in B \bar{a}_{0}$, and since $x \bar{a}_{0}=0$, we have $x \in C$. Therefore $b \in C$. Thus $C=B$.

LEMMA 1.3. Suppose $B$ is a complemented modular lattice with unit element $u, n \geqq 3$, and $a$ is a partial $n$-frame of $B$. If $x \in B$ and $u=x+\bar{a}_{0}$, then $x \sim a_{\text {; for }}$ $i=0,1, \cdots, n-1$.

Proof. (For the special case $n=3$ and $a^{*}=0$, see [1, p. 255].) Let

$$
y=\left(a_{0}+a_{i}\right)\left(x+\bar{a}_{0} \bar{a}_{i}\right) .
$$

Then

$$
y+\bar{a}_{0}=u \text { and } y \bar{a}_{0}=a_{i}\left(x+\bar{a}_{0} \bar{a}_{i}\right)=0,
$$

and hence $y \sim a_{0}$. Choosing $j<n$ with $j \neq 0$, $i$, we therefore have $y \sim a_{0} \sim a_{j}$, and since $\left(y+a_{0}\right) a_{j} \leqq\left(a_{0}+a_{i}\right) a_{j}=0$, it follows (see e.g. [8, p. 58]) that $y \sim a_{j}$. Again, since $y\left(a_{i}+a_{j}\right)=0$ and $y \sim a_{j} \sim a_{i}$, we have $y \sim a_{i}$. Finally, since $y \leqq x+\bar{a}_{0} \bar{a}_{i}$ and $\left(x, \bar{a}_{0} \bar{a}_{i}, a_{i}\right) \perp$, we have $(x+y) a_{i}=0$ and this, together with the fact that $x \sim y \sim a_{i}$, implies that $x \sim a_{i}$.

LEMмA 1.4. If the elements $a$ and $b$ of a relatively complemented modular lattice $B$ are projective, then $a$ is the sum of finitely many elements, each of which is perspective to a part of $b$.

Proof. We consider only the case in which $a$ and $b$ are connected by a sequence of two perspectivities, $a \sim c \sim b$; the general case follows by an easy induction.

Since $a c \leqq c$, we have $a c \prec b$. Choose $a_{0}$ with $a=a c \dot{+} a_{0}$. Since perspectivities are isomorphisms, we can find $b_{0} \leqq b$ and $c_{0} \leqq c$ such that $a_{0} \sim c_{0} \sim b_{0}$. Clearly $a_{0} c_{0}=0$. Letting $c_{1}=c_{0} b_{0}$ and choosing $c_{2}$ with $c_{0}=c_{1}+c_{2}$ we can find $a_{1}, a_{2}, b_{1}, b_{2}$ such that $a_{0}=a_{1} \dot{+} a_{2}, b_{0}=b_{1} \dot{+} b_{2}, a_{1} \sim c_{1} \sim b_{1}$, and $a_{2} \sim b_{2} \sim c_{2}$. Since $c_{1} \leqq b$, we have $a_{1} \prec b$. Also observe that $a_{2} c_{2}=b_{2} c_{2}=0$. Next let $a_{3}=a_{2}\left(b_{2}+c_{2}\right)$, $b_{3}=b_{2}\left(a_{2}+c_{2}\right)$ and $c_{3}=c_{2}\left(a_{2}+b_{2}\right)$, and check that $a_{3}+b_{3}=a_{3}+c_{3}=b_{3}+c_{3}$, so that $a_{3} \sim b_{3}$. Finally, choosing $a_{4}$ so that $a_{2}=a_{3} \dot{+} a_{4}$, we can find $b_{4} \leqq b_{2}$ and $c_{4} \leqq c_{2}$ such that $a_{4} \sim c_{4} \sim b_{4}$. Since $b_{4} c_{4} \leqq b_{2} c_{2}=0$ and $a_{4}\left(b_{4}+c_{4}\right) \leqq a_{4} a_{3}=0$, we have $\left(a_{4}, b_{4}, c_{4}\right) \perp$, and we infer (see e.g. $\left[8\right.$, p. 68]) that $a_{4} \sim b_{4}$. Thus $a$ is the sum of the four elements $a c, a_{1}, a_{3}$ and $a_{4}$, each of which is perspective to a part of $b$.

We conclude this section by proving the existence of large partial $n$ frames in a simple complemented modular lattice. An ideal $C$ of a modular lattice $B$ is said to be neutral provided the condition $a \sim b \in C$ always implies that $a \in C$. We recall (cf. [2, p. 125]) that if $B$ is relatively complemented, then there is a one-to-one correspondence between the congruence relations over $B$ and the neutral ideals of $B$, since the neutral ideals are precisely the kernels of congruence relations and since each congruence relation is completely determined by its kernel. 
LEMMA 1.5. Let $a$ be an element of a relatively complemented modular lattice $B$, and let $C$ be the set of all elements $c \in B$ such that $c$ is the sum of finitely many elements, each of which is perspective to a part of $a$. Then $C$ is a neutral ideal of $B$.

Proof. Clearly $C$ is closed under addition. If $b \sim c \in C$, then there exist elements $x_{0}, x_{1}, \cdots, x_{n-1} \in B$ such that

$$
c=\sum_{i<n} x_{i} \quad \text { and } \quad x_{i}<a, \quad \text { for } i=0,1, \cdots, n-1 .
$$

Therefore

$$
b=\sum_{i<n} y_{i} \quad \text { with } \quad y_{i} \sim x_{i}, \quad \text { for } i=0,1, \cdots, n-1 .
$$

By 1.4 we have $y_{i} \in C$ for all $i<n$, and hence $b \in C$.

If $b \leqq c \in C$, then (1) holds for suitable elements $x_{i}$. Let $y_{0}=0$ and

$$
y_{i}=x_{0}+x_{1}+\cdots+x_{i-1} \quad \text { for } i=1,2, \cdots, n,
$$

and for $i=0,1, \cdots, n-1$ choose $z_{i} \in B$ so that $b y_{i+1}=b y_{i}+z_{i}$. Then

$$
b=\sum_{i<n} z_{i}
$$

For each $i<n$ we have $y_{i}+x_{i}\left(y_{i}+z_{i}\right)=y_{i}+z_{i}$, and we can therefore find $v_{i} \in B$ such that $y_{i} \dot{+} z_{i}=y_{i} \dot{+} v_{i}$ and $v_{i} \leqq x_{i}\left(y_{i}+z_{i}\right)$. Thus $z_{i} \sim v_{i}<a$, and we infer by the first part of the proof that $z_{i} \in C$. Consequently $b \in C$. The set $C$ is therefore a neutral ideal of $B$.

COROLlARY 1.6. If $a$ is a nonzero element of a simple relatively complemented modular lattice $B$, then every element of $B$ is the sum of finitely many elements, each of which is perspective to a part of $a$.

THEOREM 1.7. If $n$ is a positive integer and if $B$ is a simple complemented modular lattice of dimension at least $n$, then there exists a large partial $n$-frame of $B$.

Proof. By hypothesis there exist independent nonzero elements $b_{0}, b_{1}, \cdots, b_{n-1} \in B$, and by $n-1$ successive applications of 1.6 we obtain nonzero elements $x_{1}, x_{2}, \cdots, x_{n-1}, y_{1}, y_{2}, \cdots, y_{n-1} \in B$ such that $b_{0} \geqq x_{1} \geqq x_{2}$ $\geqq \cdots \geqq x_{n-1}$, and $x_{i} \sim y_{i} \leqq b_{i}$ whenever $0<i<n$. Letting $z_{0}=x_{n-1}$ we can therefore find $z_{1}, z_{2}, \cdots, z_{n-1} \in B$ such that $z_{0} \sim z_{i} \leqq y_{i}$ for $i=1,2, \cdots, n-1$. Inasmuch as $\left(z_{0}, z_{1}, \cdots, z_{n-1}\right) \perp$, there exists a partial $n$-frame $a$ of $B$ such that $a_{i}=z_{i}$ for $i=0,1, \cdots, n-1$, and using 1.6 we conclude that $a$ is large.

2. Complemented Arguesian lattices. A lattice $B$ is said to be ARguEsian if and only if it satisfies the following condition: For all $a_{0}, a_{1}, a_{2}, b_{0}, b_{1}, b_{2} \in B$, if 


$$
y=\left(a_{0}+a_{1}\right)\left(b_{0}+b_{1}\right)\left[\left(a_{0}+a_{2}\right)\left(b_{0}+b_{2}\right)+\left(a_{1}+a_{2}\right)\left(b_{1}+b_{2}\right)\right],
$$

then

$$
\left(a_{0}+b_{0}\right)\left(a_{1}+b_{1}\right)\left(a_{2}+b_{2}\right) \leqq a_{0}\left(a_{1}+y\right)+b_{0}\left(b_{1}+y\right) .
$$

The name is suggested by the fact (cf. [6]) that a projective geometry satisfies Desargues' Law if and only if the lattice of all its subspaces is Arguesian. It is known (cf. [6]) that every lattice of commuting equivalence relations is Arguesian and, a fortiori, so is every coordinatizable lattice.

LemMa 2.1. Every complemented modular lattice $B$ which possesses a large partial $n$-frame a with $n \geqq 4$ is Arguesian.

Proof. By [3], $B$ can be mapped isomorphically into the lattice $A$ of all subspaces of a projective space, the unit element $u$ of $B$ being mapped onto the whole space. We may assume that $B$ is actually a sublattice of $A$. The space $u$ can be decomposed into pairwise disjoint subspaces $u_{i}, i \in I$, in such a way that the line determined by two distinct points is nondegenerate if and only if both points belong to the same component. By hypothesis, the unit element $u$ of $A$ has the property that it is the sum of finitely many elements, each of which is perspective to a part of $a_{0}$, and it follows by 1.5 that every element of $A$ has this property. In particular, every point of $u$ is perspective to a point in $a_{0}$. Now consider any $i \in I$, and choose a point $p$ in $u_{i}$. Then $p \sim q_{0}$ for some point $q_{0}$ is $a_{0}$. For $j=1,2,3$ we have $a_{0} \sim a_{j}$, and we can therefore find a point $q_{j}$ in $a_{j}$ such that $q_{0} \sim q_{j}$. Since $p$ is in $u_{i}$, the points $q_{0}, q_{1}, q_{2}, q_{3}$ must also be in $u_{i}$, and since these four points are independent, the dimension of $u_{i}$ is at least three. Being nondegenerate, $u_{i}$ must therefore satisfy Desargues' Law. Consequently the whole space $u$ satisfies Desargues' Law, whence it follows that $A$ is Arguesian, and therefore $B$ is also Arguesian.

LEMMA 2.2. In a simple relatively complemented modular lattice $B$ every nontrivial ideal is simple.

Proof. Suppose $C$ is a nontrivial ideal of $B$, and let $\equiv$ be a congruence relation over $C$. If $\equiv$ is not the identity, then there exists a nonzero element $a \in C$ such that $a \equiv 0$. For any $c \in B$ there exist, by 1.6 , elements $x_{i} \in B, i=0$, $1, \cdots, n-1$, with

$$
c=\sum_{i<n} x_{i}
$$

and $x_{i}<a$ in $B$ for all $i<n$. If $c \in C$, then for each $i<n$ we have $x_{i} \in C$ and $x_{i}<a$ in $C$. We conclude that $x_{i} \equiv 0$ for each $i<n$, and therefore $c \equiv 0$. Thus $C$ is simple.

TheOREM 2.3. Every simple relatively complemented modular lattice $B$ of dimension four or more is Arguesian. 
Proof. For the complemented case this follows from 1.7 and 2.1. In the general case we observe that any six elements $a_{0}, a_{1}, a_{2}, b_{0}, b_{1}, b_{2} \in B$ are contained in a principal ideal $B u$ whose dimension is at least four. Since, by 2.2 , the complemented modular lattice $B u$ is simple, it follows that the elements $a_{i}, b_{i}$ must satisfy the Arguesian condition. Consequently $B$ is Arguesian.

3. The embedding theorem. Our principal result will be based on the following embedding theorem:

ThEOREM 3.1. If $a$ is a partial three-frame of a complemented Arugesian lattice $B$, then there exist a complemented Arguesian lattice $B^{\prime}$ and an element $b \in B^{\prime}$ such that

(i) $B$ is an ideal of $B^{\prime}$ and $B^{\prime}=B \dot{+} B^{\prime} b$.

(ii) $b \sim a_{0}$ in $B^{\prime}$.

As will be shown, this theorem is an easy consequence of

TheOREm 3.2. Suppose $A^{\prime}$ is the lattice of all subgroups of an Abelian group $u^{\prime}, u$ is a subgroup of $u^{\prime}$, and $B$ is a complemented sublattice of the lattice $A$ of all subgroups of $u$. If $a$ is a partial four-frame of $A^{\prime}$ such that $\bar{a}_{0}=u$ and $a_{1}, a_{2}, a_{3}$, $a_{1,2}, a_{1,3}, a^{*} \in B$, then the sublattice $B^{\prime}$ of $A^{\prime}$ which is generated by the set $B \cup\left\{a_{0}, a_{0,1}\right\}$ is complemented, and $B$ is an ideal of $B^{\prime}$.

Proof that Theorem 3.1 follows from Theorem 3.2. Since the lattice $B$ in Theorem 3.1 is complemented and Arguesian, it is isomorphic to a sublattice of the lattice $A$ of all subgroups of an Abelian group $u$. It may therefore be assumed that $B$ is actually a sublattice of $A$, and that $u \in B$. Since the groups $a_{0}, a_{1}, a_{2}$ are perspective, they are isomorphic. Let $u^{\prime}$ be an Abelian group that is a direct product of $u$ and of a group $b$ that is isomorphic to the groups $a_{i}$, and let $A^{\prime}$ be the lattice of all subgroups of $u^{\prime}$.

If $\phi$ is an isomorphism of $b$ onto $a_{0}$, then the set $x$ of all elements of the form $\alpha-\phi(\alpha)$ with $\alpha \in b$ is a subgroup of $u^{\prime}$ and the axis of a perspectivity between $b$ and $a_{0}$. By the remark that follows Definition 1.1 we infer that there exists a partial four-frame $c$ of $A^{\prime}$ such that $c_{0}=b, c_{i}=a_{i-1}$ for $i=1,2,3$, $c_{0,1}=x, c_{i, j}=a_{i-1, j-1}$ for $i, j=1,2,3$, and $c^{*}=a^{*}$. Thus the hypothesis of Theorem 3.2 is satisfied with $a$ replaced by $c$, and the sublattice $B^{\prime}$ of $A^{\prime}$ which is generated by the set $B \cup\{b, x\}$ is complemented and contains $B$ as an ideal. Inasmuch as $u^{\prime}=u \dot{+} b$, it follows that $B^{\prime}=B \dot{+} B^{\prime} b$ and, finally, since $x \in B^{\prime}$ we have $b \sim a_{0}$ in $B^{\prime}$.

Reduction of the proof of Theorem 3.2. In the discussion that follows, Theorem 3.2 will be shown to follow from four statements, (1)-(4), whose validity will be established in \$\$4-7.

The group $u^{\prime}$ is the direct product of its subgroups $a_{0}, a_{1}, a_{2}, a_{3}$ and $a^{*}$, and since the groups $a_{i}$ and $a_{j}$ are perspective, they are isomorphic. In fact, each element $\alpha_{i} \in a_{i}$ can be uniquely written in the form $\alpha_{i}=\alpha_{j}+\beta$ with $\alpha_{j} \in a_{j}$ and $\beta \in a_{i, j}$, and the mapping $\alpha_{i} \rightarrow \alpha_{j}$ is an isomorphism of $a_{i}$ onto $a_{j}$. 
If $\lambda$ is a permutation of the set $\{0,1,2,3\}$, and if we map each $a_{i}$ in this manner onto $a_{\lambda(i)}$, and map each element of $a^{*}$ onto itself, then these mappings permute the factors of $u^{\prime}$ and therefore induce an automorphism $a^{\lambda}$ of $u^{\prime}$. Alternatively, $a^{\lambda}$ can be characterized by the conditions that $a^{\lambda}(\alpha)=\alpha$ whenever $\alpha \in a^{*}$ and, for all $\alpha \in a_{i}, i=0,1,2,3$,

$$
a^{\lambda}(\alpha) \in a(i) \text { and } \alpha-a^{\lambda}(\alpha) \in a_{i, \lambda(i)} .
$$

The automorphism of $A^{\prime}$ that is induced by $a^{\lambda}$ will be denoted by the same symbol $a^{\lambda}$, and if $X$ is any subset of $A^{\prime}$, then $a^{\lambda}(X)$ will be the set consisting of the images of all the elements of $X$.

If $i$ and $j$ are distinct elements of the set $\{0,1,2,3\}$, let $(i, j)$ be the permutation that interchanges $i$ and $j$, leaving the other two members of the set fixed. It will be shown (Lemma 4.3) that

$$
a^{(i, j)}(x)=\left(x+a_{i, j}\right) \bar{a}_{j} \quad \text { whenever } \quad \bar{a}_{i} \geqq x \in A^{\prime} .
$$

Since the elements $a_{0, j}=\left(a_{0,1}+a_{1, j}\right)\left(a_{0}+a_{j}\right)$ belong to $B^{\prime}$, it follows that the lattices $B_{j}(a)=a^{(0, j)}(B), j=1,2,3$, are sublattices of $B^{\prime}$. For $i, j=1,2,3$ with $i \neq j$ let $B_{i, j}(a)$ be the set of all elements $b$ of the form

$$
b=x+\left(y+a_{i}\right)\left(z+a_{j}\right)
$$

with $x \in B, y \in B_{i}(a), z \in B_{j}(a)$ and $y u=z u=0$. In particular, let $B(a)=B_{1,2}(a)$. It turns out (Lemma 5.2) that $B_{i, j}(a)$ is actually independent of $i$ and $j$. That is, the formula

$$
B(a)=B_{i, j}(a)
$$

holds for $i, j=1,2,3$ with $i \neq j$.

Clearly $B \cup\left\{a_{0}, a_{0,1}\right\} \subseteq B(a) \subseteq B^{\prime}$. After a rather lengthy argument it will be shown (Theorem 7.4) that

$$
B(a) \text { is a complemented sublattice of } A^{\prime} \text {. }
$$

Consequently $B(a)=B^{\prime}$, and $B^{\prime}$ is complemented. Finally, it is easy to prove (Theorem 7.4 again) that

$$
B \text { is an ideal of } B(a) \text {, }
$$

thereby completing the proof of our theorem.

Thus the proof of Theorem 3.2 has been reduced to showing that the statements (1)-(4) hold. The next four sections will be devoted to this task. In the proof of (3) we shall have occasions to consider, not one fixed partial four-frame, but several different ones. It is for this reason that the notation introduced above has been so chosen that it reflects the dependence of the various constructions upon the frame.

For convenience we collect here the various assumptions and notations that will be used throughout the next four sections. 
Sectional assumptions 3.3. Throughout $\$ \S 4-7$ the following assumptions and notations will be in effect:

(i) $A^{\prime}$ is the lattice of all subgroups of an Abelian group $u^{\prime}$.

(ii) $A$ is the lattice of all subgroups of a subgroup $u$ of $u^{\prime}$.

(iii) $B$ is a complemented sublattice of $A$ with $u \in B$.

(iv) $F$ is the set of all partial four-frames $a$ of $A^{\prime}$ such that $\bar{a}_{0}=u$ and $a_{1}, a_{2}, a_{3}, a_{1,2}, a_{1,3}, a^{*} \in B$.

(v) $Q$ is the set of all permutations of the set $\{0,1,2,3\}$.

(vi) For each $a \in F$ and $\lambda \in Q, a^{\lambda}$ is the unique automorphism of $u^{\prime}$ such that $a^{\lambda}(\alpha)=\alpha$ whenever $\alpha \in a^{*}$ and, for all $\alpha \in a_{i}, i=0,1,2,3$,

$$
a^{\lambda}(\alpha) \in a_{\lambda(i)} \text { and } \alpha-a^{\lambda}(\alpha) \in a_{i, \lambda(i)} .
$$

(vii) For all $a \in F, B_{0}(a)=B$ and $B_{i}(a)=a^{(0, i)}(B)$ for $i=1,2,3$.

(viii) For all $a \in F$ and for $i, j=1,2,3$ with $i \neq j, B_{i, j}(a)$ is the set of all elements $b \in A^{\prime}$ of the form

$$
b=x+\left(y+a_{i}\right)\left(z+a_{j}\right)
$$

with $x \in B, y \in B_{i}(a), z \in B_{j}(a)$ and $y u=z u=0$.

(ix) For all $a \in F, B(a)=B_{1,2}(a)$.

4. The automorphisms $a^{\lambda}$.

Lemma 4.1. If $a \in F$ and $\lambda, \mu \in Q$, then $a^{\lambda} a^{\mu}=a^{\lambda \mu}$.

Proof. Suppose $i$ is one of the integers $0,1,2,3$, and let $j=\mu(i)$ and $k=\lambda(j)$. Given $\alpha \in a_{i}$, let $\beta=a^{\mu}(\alpha)$ and $\gamma=a^{\lambda}(\beta)$. Then

$$
\beta \in a_{j}, \quad \alpha-\beta \in a_{i, j}, \quad \gamma \in a_{k}, \quad \beta-\gamma \in a_{j, k} .
$$

Consequently

$$
\alpha-\gamma \in\left(a_{i}+a_{k}\right)\left(a_{i, j}+a_{j, k}\right)=a_{i, k},
$$

so that $\gamma=a^{\lambda \mu}(\alpha)$. Thus the automorphisms $a^{\lambda \mu}$ and $a^{\lambda} a^{\mu}$ agree on each of the groups $a_{i}$, and since they both map each member of $a^{*}$ onto itself, they must be identical.

Lemma 4.2. For all $a \in F$, and for $i, j=0,1,2,3$,

$$
a^{(i, j)}(\alpha)=\alpha \text { whenever } \alpha \in \bar{a}_{i} \bar{a}_{j} .
$$

Proof. Let $\lambda=(i, j)$. If $i, j \neq k=0,1,2,3$, then $a_{k, \lambda(k)}=0$, and it follows by 3.3(vi) that $a^{\lambda}(\alpha)=\alpha$ for all $\alpha \in a_{k}$. Since $a^{\lambda}$ also maps each member of $a^{*}$ onto itself, the conclusion follows.

Lemмa 4.3. For all $a \in F$, and for $i, j=0,1,2,3$,

$$
a^{(i, j)}(x)=\left(x+a_{i, j}\right) \bar{a}_{j} \quad \text { whenever } \quad \bar{a}_{i} \geqq x \in A^{\prime} .
$$

Proof. Given $\alpha \in x$, there exist $\beta, \gamma$ such that 


$$
\beta \in a_{j}, \quad \alpha-\beta \in \bar{a}_{i} \bar{a}_{j}, \quad \gamma \in a_{i}, \quad \beta-\gamma \in a_{i, j} .
$$

Using 4.2 and 3.3 (vi) we infer that

$$
a^{(i, j)}(\alpha)=a^{(i, j)}(\alpha-\beta)+a^{(i, j)}(\beta)=\alpha-\beta+\gamma .
$$

Consequently $a^{(i, j)}(\alpha)$ belongs to the group

$$
\left(x+a_{i, j}\right)\left(a_{i}+\bar{a}_{i} \bar{a}_{j}\right)=\left(x+a_{i, j}\right) \bar{a}_{j} .
$$

Conversely, if $\delta$ belongs to this group, then there exist $\alpha$ such that $\alpha \in x$ and $\delta-\alpha \in a_{i, j}$. Choosing $\beta$ and $\gamma$ so that (1) holds, observe that since the elements $\beta-\gamma$ and $\delta-\alpha$ belong to $a_{i, j}$ and the elements $\delta, \alpha-\beta$ and $\gamma$ belong to $\bar{a}_{j}$, the element $\delta-\alpha+\beta-\gamma$ belongs to both $a_{i, j}$ and $\bar{a}_{j}$. Since $a_{i, j} \bar{a}_{j}=0$, we conclude that

$$
\delta=\alpha-\beta+\gamma=a^{(i, j)}(\alpha) \in a^{(i, j)}(x) .
$$

We remark that from now on no use will be made of the fact that the elements of $A^{\prime}$ are groups and that the lattice multiplication and inclusion coincide with the set-theoretic intersection and inclusion. It is for this reason that we have used the notation employed when speaking about lattices in general rather than the customary set-theoretic symbols $\cap$ and $\subseteq$.

Lemma 4.4. If $a \in F, \lambda \in Q$ and $\lambda(0)=0$, then $a^{\lambda}(B)=B$.

Proof. We need only consider the case in which $\lambda$ is a transposition, $\lambda=(i, j)$. Let $k$ be the remaining one of the three integers $1,2,3$. Given $b \in B$, let $x=b\left(a_{k}+a^{*}\right)$ and choose $y, z \in B$ so that

$$
b=x+y+z, \quad y \leqq \bar{a}_{i}, \quad z \bar{a}_{i}=0 .
$$

Let

$$
v=\left(z+a_{k}+a^{*}\right)\left(a_{i}+a_{j}\right), \quad w=\left(z+a_{j}\right) \bar{a}_{j} .
$$

Then

$$
\left(v+a_{k}+a^{*}\right)\left(w+a_{j}\right)=\left(z+a_{k}+a^{*}\right)\left(z+a_{j}\right)=z,
$$

because $\left(a_{j}, a_{k}, a^{*}, z\right) \perp$. By 4.2 every element contained in $\bar{a}_{i} \bar{a}_{j}$ is mapped onto itself by $\lambda$, hence in particular this is true of $x, a_{k}$ and $a^{*}$. Also, by 4.3, $a_{i}$ and $a_{j}$ are mapped onto each other, and $y$ and $w$ are mapped onto elements belonging to $B$. By 4.1 ,

$$
a^{(i, j)}=a^{(j, k)} a^{(i, j)} a^{(i, k)},
$$

and by successive applications of 4.3 we see that $a^{(i, k)}$ maps $v$ onto an element $v^{\prime} \in B$ which is contained in $a_{k}+a_{j}$, that $a^{(i, j)}$ maps $v^{\prime}$ onto an element $v^{\prime \prime} \in B$ contained in $a_{k}+a_{i}$, and $a^{(j, k)}$ maps $v^{\prime \prime}$ onto an element $v^{\prime \prime \prime} \in B$ which is contained in $a_{j}+a_{i}$. Thus $a^{\lambda}(v)=v^{\prime \prime \prime}$ is a member of $B$. It now follows from (2) and (1) that $a^{\lambda}(z) \in B$ and $a^{\lambda}(b) \in B$. Thus $a^{\lambda}$ maps $B$ into itself, and the fact 
that $a^{\lambda}$ is of order two enables us to conclude that $a^{\lambda}(B)=B$.

Lemma 4.5. For all $a \in F$, and for $i, j=0,1,2,3, a^{(i, j)} \operatorname{maps} B_{i}(a)$ isomorphically onto $B_{j}(a)$.

Proof. If $i=0$ or $j=0$, then this follows from 3.3 (vi), (vii). The case $i=j$ is trivial since $a^{(i, i)}$ is the identity automorphism of $u^{\prime}$. If $i \neq j \neq 0 \neq i$, then

$$
a^{(i, j)}=a^{(i, 0)} a^{(i, j)} a^{(0, i)},
$$

$a^{(0, i)}$ maps $B_{i}(a)$ isomorphically onto $B, a^{(i, j)}$ maps $B$ isomorphically onto $B$, and $a^{(i, 0)}$ maps $B$ isomorphically onto $B_{j}(a)$.

LeммA 4.6. For all $a \in F$, and for $i, j, k=0,1,2,3$ with $k \neq i, j, a^{(i, j)}$ maps $B_{k}(a)$ isomorphically onto itself.

Proof. We may assume that $i \neq j$. If $k=0$, then the conclusion follows from 4.4. If $i, j, k \neq 0$, then

$$
a^{(i, j)}=a^{(0, k)} a^{(i, j)} a^{(0, k)},
$$

and under the mappings on the right $B_{k}(a)$ is first taken into $B$, then into $B$, and finally into $B_{k}(a)$.

If either $i$ or $j$ is equal to 0 , say $j=0$, we have

$$
a^{(i, j)}\left(B_{k}(a)\right)=a^{(i, 0)}\left(B_{k}(a)\right)=a^{(i, 0)} a^{(0, k)}(B)=a^{(i, 0)} a^{(0, k)} a^{(i, k)}(B),
$$

where in the last step we use 4.4 . Since $(i, 0)(0, k)(i, k)=(0, k)$, we conclude with the aid of 4.1 that

$$
a^{(i, j)}\left(B_{k}(a)\right)=a^{(0, k)}(B)=B_{k}(a) .
$$

Lemмa 4.7. For all $a \in F$ and $\lambda \in Q$, and for $k=0,1,2,3$,

$$
a^{\lambda}\left(B_{k}(a)\right)=B_{\lambda(k)}(a) .
$$

Proof. If $\lambda(k)=k$, then this follows from 4.1 and 4.6. If $\lambda(k) \neq k$, then we let $\mu=(k, \lambda(k))$ and $\nu=\mu \lambda$. Since $\lambda=\mu \nu$ and $\nu(k)=k$, we conclude with the aid of 4.1 and 4.5 that

$$
a^{\lambda}\left(B_{k}(a)\right)=a^{\mu}\left(a^{\nu}\left(B_{k}(a)\right)=a^{\mu}\left(B_{k}(a)\right)=B_{\lambda(k)}(a) .\right.
$$

Lemмa 4.8. Suppose $a \in F$ and $i, j=0,1,2,3$. If $x \in A^{\prime}$ and $x \leqq \bar{a}_{i} \bar{a}_{j}$, then the conditions $x \in B_{i}(a)$ and $x \in B_{j}(a)$ are equivalent.

Proof. By $4.2, a^{(i, j)}(x)=x$. Hence the conclusion follows by 4.7 .

5. Alternative characterizations of $B(a)$.

Lemma 5.1. Suppose $a \in F, i, j=1,2,3, i \neq j, b \in A^{\prime}$, and $b u=0$. If we let 


$$
y=\left(b+a_{i}\right) \bar{a}_{i}, \quad z=\left(b+a_{j}\right) \bar{a}_{j}
$$

then

(i) $b=\left(y+a_{i}\right)\left(z+a_{j}\right)$,

(ii) $b \in B_{i, j}(a)$ if and only if $y \in B_{i}(a)$ and $z \in B_{j}(a)$.

Proof. We have

$$
y+a_{i}=b+a_{i}, \quad z+a_{j}=b+a_{j},
$$

and since $\left(a_{i}, a_{j}, b\right) \perp$, it follows that

$$
\left(\mathrm{y}+a_{i}\right)\left(z+a_{j}\right)=\left(b+a_{i}\right)\left(b+a_{j}\right)=b .
$$

If $b \in B_{i, j}(a)$, then it follows from 3.3 (viii) that there exist $y^{\prime}, z^{\prime}$ such that

$$
\begin{gathered}
b=\left(y^{\prime}+a_{i}\right)\left(z^{\prime}+a_{j}\right), \\
y^{\prime} \in B_{i}(a), \quad z^{\prime} \in B_{j}(a), \quad y^{\prime} u=z^{\prime} u=0 .
\end{gathered}
$$

Let

$$
x=\left(z^{\prime}+a_{i}\right) \bar{a}_{i}, \quad y^{\prime \prime}=y^{\prime}\left(x+a_{j}\right) .
$$

Since $x \leqq \bar{a}_{j}$ we have

$$
x=\left(z^{\prime}+a_{i}\right) \bar{a}_{i} \bar{a}_{j}
$$

and inasmuch as $z^{\prime}+a_{i}$ and $\bar{a}_{i} \bar{a}_{j}$ belong to $B_{j}(a)$, this implies that $x \in B_{j}(a)$ and therefore, by $4.8, x \in B_{i}(a)$. Consequently $y^{\prime \prime} \in B_{i}(a)$. Furthermore

$$
\begin{aligned}
y^{\prime \prime}+a_{i} & =y^{\prime}\left[\left(z^{\prime}+a_{i}\right) \bar{a}_{i}+a_{j}\right]+a_{i} \\
& =y^{\prime}\left(z^{\prime}+a_{i}+a_{j}\right) \bar{a}_{i}+a_{i} \\
& =y^{\prime}\left(z^{\prime}+a_{i}+a_{j}\right)+a_{i} \\
& =\left(y^{\prime}+a_{i}\right)\left(z^{\prime}+a_{i}+a_{j}\right) \\
& =\left(y^{\prime}+a_{i}\right)\left(z^{\prime}+a_{j}\right)+a_{i}=b+a_{i},
\end{aligned}
$$

and since $y^{\prime \prime} \leqq \bar{a}_{i}$ we have

$$
y^{\prime \prime}=\left(y^{\prime \prime}+a_{i}\right) \bar{a}_{i}=\left(b+a_{i}\right) \bar{a}_{i}=y .
$$

Therefore $y \in B_{i}(a)$. Similarly $z \in B_{j}(a)$, and the forward implication in (ii) holds. Since

$$
y \bar{a}_{0}=\left(b+a_{i}\right) \bar{a}_{i} \bar{a}_{0}=\left(b \bar{a}_{0}+a_{i}\right) \bar{a}_{i}=a_{i} \bar{a}_{i}=0
$$

and similarly $z \bar{a}_{0}=0$, the backward implication follows from (i) and 3.3 (viii).

Lemma 5.2. If $a \in F, i, j=1,2,3$, and $i \neq j$, then $B_{i, j}(a)=B(a)$.

Proof. Since $B_{i, j}(a)=B_{j, i}(a)$, it suffices to show that if $i, j, k=1,2,3$ and $i \neq j \neq k \neq i$, then $B_{i, j}(a)=B_{i, k}(a)$. In order to prove this we need only show that if $b \in B_{i, j}(a)$ and $b u=0$, then $b \in B_{i, k}(a)$. 
Let

$$
y_{p}=\left(b+a_{p}\right) \bar{a}_{p} \quad \text { for } p=1,2,3 .
$$

By 5.1, $y_{i} \in B_{i}(a)$ and $y_{j} \in B_{j}(a)$, and the problem reduces to showing that $y_{k} \in B_{k}(a)$. Let

$$
\begin{aligned}
& z_{p}=\left(y_{p}+a_{k}\right) \bar{a}_{k} \quad \text { for } p=i, j, \\
& y^{\prime}=\left(z_{i}+a_{i}\right)\left(z_{j}+a_{j}\right) .
\end{aligned}
$$

Since $z_{i} \leqq \bar{a}_{i}$, we have $z_{i}=\left(y_{i}+a_{k}\right) \bar{a}_{i} \bar{a}_{k} \in B_{i}(a)$. Similarly $z_{j} \in B_{j}(a)$. Furthermore, both $z_{i}$ and $z_{j}$ are contained in $\bar{a}_{k}$, whence we infer by 4.8 that $z_{i}, z_{j}$ $\in B_{k}(a)$. Consequently $y^{\prime} \in B_{k}(a)$. Next observe that

$$
\begin{aligned}
y^{\prime}+a_{k} & =\left(z_{i}+a_{i}\right)\left(z_{j}+a_{j}\right)+a_{k} \\
& =\left(z_{i}+a_{i}+a_{k}\right)\left(z_{j}+a_{j}+a_{k}\right),
\end{aligned}
$$

because $z_{i}, z_{j}, a_{i}$ and $a_{j}$ are contained in $\bar{a}_{k}$, and the elements $z_{i}+a_{i}, z_{j}+a_{j}$ and $a_{k}$ therefore generate a distributive lattice. For $p=i, j$ we have

$$
z_{p}+a_{k}=y_{p}+a_{k}, \quad y_{p}+a_{p}=b+a_{p} .
$$

Consequently

$$
y^{\prime}+a_{k}=\left(b+a_{i}+a_{k}\right)\left(b+a_{j}+a_{k}\right)=b+a_{k},
$$

because $\left(a_{i}, a_{j}, a_{k}, b\right) \perp$. Using the fact that $y^{\prime} \leqq \bar{a}_{k}$, we conclude that

$$
y^{\prime}=\left(y^{\prime}+a_{k}\right) \bar{a}_{k}=\left(b+a_{k}\right) \bar{a}_{k}=y_{k},
$$

so that $y_{k} \in B_{k}(a)$ as was to be shown.

Lemma 5.3. If $a \in F, i=0,1,2,3, b \in A^{\prime}$, and $b \leqq \bar{a}_{i}$, then the conditions $b \in B(a)$ and $b \in B_{i}(a)$ are equivalent.

Proof. The case $i=0$ being obvious, we assume that $i>0$. First suppose $b \in B_{i}(a)$. Since $B_{i}(a)$ is a complemented sublattice of $A^{\prime}$, and since

$$
b u=b \bar{a}_{0}=b \bar{a}_{0} \bar{a}_{i} \in B_{i}(a),
$$

there exists $c \in B_{i}(a)$ such that $b=b u+c$. By $4.8, b u \in B$. Also

$$
\left(c+a_{i}\right) \bar{a}_{i}=c \in B_{i}(a),
$$

and if we choose $j=1,2,3$ with $j \neq i$, then the element

$$
\left(c+a_{j}\right) \bar{a}_{j}=\left(c+a_{j}\right) \bar{a}_{i} \bar{a}_{j}
$$

belongs to $B_{i}(a)$ and therefore, by 4.8 , to $B_{j}(a)$. Thus we infer with the aid of 5.1 and 5.2 that $c \in B(a)$, and therefore that $b \in B(a)$.

Conversely, if $b \in B(a)$ then $b u \in B$ and we can find $c \in B(a)$ such that $b=b u+c$. Observing that $\left(c+a_{i}\right) \bar{a}_{i}=c$, we use 5.1 and 5.2 to infer that $c \in B_{i}(a)$. But $b u \in B_{i}(a)$ by 4.8 , and we therefore conclude that $b \in B_{i}(a)$. 
Lemma 5.4. Suppose $a \in F, i=1,2,3, b \in A^{\prime}$, and $b u=0$. If $b \in B(a)$, and if we let then

(i) $y=\left(b+a_{i}\right) \bar{a}_{i}, z=\left(b+\bar{a}_{0} \bar{a}_{i}\right)\left(a_{0}+a_{i}\right)$,

(ii) $b=\left(y+a_{i}\right)\left(z+\bar{a}_{0} \bar{a}_{i}\right)$,

(iii) $y \in B_{i}(a), z \in B(a), z \leqq a_{0}+a_{i}, y u=z u=0$.

Conversely, if there exist $y, z \in A^{\prime}$ which satisfy (ii) and (iii), then $b \in B(a)$.

Proof. Let $j, k=1,2,3$ with $i \neq j \neq k \neq i$. If $b \in B(a)$, and if $y$ and $z$ are defined by (i), then it follows from 5.1 and 5.2 that $y \in B_{i}(a)$ and that the element

$$
x=\left(b+a_{j}\right) \bar{a}_{j}
$$

is a member of $B_{j}(a)$. It is also easy to check that

$$
z=\left(x+a_{k}+a^{*}\right)\left(a_{0}+a_{i}\right),
$$

and we therefore have $z \in B_{j}(a)$ and consequently, by $5.3, z \in B(a)$. Next observe that

$$
\begin{aligned}
& y u=\left(b+a_{i}\right) \bar{a}_{0} \bar{a}_{i}=\left(b \bar{a}_{0}+a_{i}\right) \bar{a}_{i}=a_{i} \bar{a}_{i}=0, \\
& z u=\left(b+\bar{a}_{0} \bar{a}_{i}\right)\left(a_{0}+a_{i}\right) \bar{a}_{0}=\left(b+\bar{a}_{0} \bar{a}_{i}\right) a_{i}=0,
\end{aligned}
$$

the last equality holding because $\left(\bar{a}_{0} \bar{a}_{i}, a_{i}, b\right) \perp$. Thus (iii) holds. Also,

$$
y+a_{i}=b+a_{i}, \quad z+\bar{a}_{0} \bar{a}_{i}=b+\bar{a}_{0} \bar{a}_{i},
$$

and again using the fact that $\left(\bar{a}_{0} \bar{a}_{i}, a_{i}, b\right) \perp$ we infer that (ii) holds.

Conversely, suppose (ii) and (iii) hold. Let

$$
\begin{aligned}
y^{\prime} & =y\left(z+\bar{a}_{0}\right), & z^{\prime} & =z\left(y+\bar{a}_{0}\right), \\
x & =\left(y^{\prime}+a_{j}\right) \bar{a}_{i} \bar{a}_{j}, & v & =\left(x+a_{i}\right)\left(z^{\prime}+a_{k}+a^{*}\right), \\
y^{\prime \prime} & =\left(y+\bar{a}_{0} \bar{a}_{i}\right) a_{0}, & z^{\prime \prime} & =\left(z+a_{i}\right) a_{0} .
\end{aligned}
$$

Observe that $z+a_{i}, a_{0}$ and $\vec{a}_{0} \bar{a}_{i}$ generate a distributive lattice because the first two of these three elements are contained in $a_{0}+a_{i}$, which is disjoint from $\bar{a}_{0} \bar{a}_{i}$. Therefore

$$
\begin{aligned}
z^{\prime \prime}+\bar{a}_{0} \bar{a}_{i} & =\left(z+a_{i}\right) a_{0}+\bar{a}_{0} \bar{a}_{i} \\
& =\left(z+a_{i}+\bar{a}_{0} \bar{a}_{i}\right)\left(a_{0}+\bar{a}_{0} \bar{a}_{i}\right)=\left(z+\bar{a}_{0}\right) \bar{a}_{i}, \\
y^{\prime}=y\left(z+\bar{a}_{0}\right) & =y \bar{a}_{i}\left(z+\bar{a}_{0}\right)=y\left(z^{\prime \prime}+\bar{a}_{0} \bar{a}_{i}\right) .
\end{aligned}
$$

Now $z, a_{i}$ and $a_{0}$ are members of $B_{j}(a)$, so that $z^{\prime \prime} \in B_{j}(a)$. Consequently $z^{\prime \prime} \in B_{i}(a)$ by 4.8 . Since $y$ and $\bar{a}_{0} \bar{a}_{i}$ are also members of $B_{i}(a)$, it follows that $y^{\prime} \in B_{i}(a)$. A similar argument will show that $z^{\prime} \in B_{j}(a)$. In fact, since $y+\bar{a}_{0} \bar{a}_{i}$ and $a_{0}$ are contained in the element $\bar{a}_{i}$, which is disjoint from $a_{i}$, we have 


$$
\begin{aligned}
y^{\prime \prime}+a_{i} & =\left(y+\bar{a}_{0} \bar{a}_{i}\right) a_{0}+a_{i} \\
& =\left(y+\bar{a}_{0} \bar{a}_{i}+a_{i}\right)\left(a_{0}+a_{i}\right)=\left(y+\bar{a}_{0}\right)\left(a_{0}+a_{i}\right), \\
z^{\prime}=z\left(y+\bar{a}_{0}\right) & =z\left(a_{0}+a_{i}\right)\left(y+\bar{a}_{0}\right)=z\left(y^{\prime \prime}+a_{i}\right),
\end{aligned}
$$

and observing that $y^{\prime \prime} \in B_{i}(a)$, hence $y^{\prime \prime} \in B_{j}(a)$, we conclude that $z^{\prime} \in B_{j}(a)$. Furthermore, from the fact that $y^{\prime} \in B_{i}(a)$ we readily infer that $x \in B_{i}(a)$, hence $x \in B_{j}(a)$, and this together with the fact that $z^{\prime} \in B_{j}(a)$ yields $v \in B_{j}(a)$.

Since $y^{\prime} u=0$ and

$$
v u=\left(x+a_{i}\right)\left(z+a_{k}+a^{*}\right) \bar{a}_{0}=\left(x+a_{i}\right)\left(a_{k}+a^{*}\right)=0,
$$

we see by 5.1 and 5.2 that the proof will be completed if we show that

$$
y^{\prime}=\left(b+a_{i}\right) \bar{a}_{i}, \quad v=\left(b+a_{j}\right) \bar{a}_{j} .
$$

Since $y^{\prime} \leqq \bar{a}_{i}$, the first equation follows from the fact that

$$
\begin{aligned}
b+a_{i} & =\left(y+a_{i}\right)\left(z+\bar{a}_{0} \bar{a}_{i}\right)+a_{i}=\left(y+a_{i}\right)\left(z+\bar{a}_{0}\right) \\
& =y\left(z+\bar{a}_{0}\right)+a_{i}=y^{\prime}+a_{i} .
\end{aligned}
$$

To prove the second equation in (1) observe that

$$
\begin{aligned}
x+a_{k}+a^{*} & =\left(y^{\prime}+\bar{a}_{0} \bar{a}_{i}\right) \bar{a}_{j}, \\
v+a_{k}+a^{*} & =\left(x+a_{i}+a_{k}+a^{*}\right)\left(z^{\prime}+a_{k}+a^{*}\right) \\
& =\left[\left(y^{\prime}+\bar{a}_{0} \bar{a}_{i}\right) \bar{a}_{j}+a_{i}\right]\left(z^{\prime}+a_{k}+a^{*}\right) .
\end{aligned}
$$

In the last product the second factor is included in the first because

$$
\begin{aligned}
& z^{\prime} \leqq\left(y+\bar{a}_{0}\right)\left(z+\bar{a}_{0}\right)=y\left(z+\bar{a}_{0}\right)+\bar{a}_{0}=y^{\prime}+\bar{a}_{0}, \\
& z^{\prime} \leqq\left(y^{\prime}+\bar{a}_{0}\right) \bar{a}_{j}=\left(y^{\prime}+\bar{a}_{0} \bar{a}_{i}\right) \bar{a}_{j}+a_{i} .
\end{aligned}
$$

Consequently

$$
\begin{aligned}
v+a_{k}+a^{*} & =z^{\prime}+a_{k}+a^{*}, \\
v+\bar{a}_{0} \bar{a}_{i} & =z^{\prime}+\bar{a}_{0} \bar{a}_{i}=\left(z+\bar{a}_{0} \bar{a}_{i}\right)\left(y+\bar{a}_{0}\right) \\
& =\left(z+\bar{a}_{0} \bar{a}_{i}\right)\left(y+a_{i}\right)+\bar{a}_{0} \bar{a}_{i}=b+\bar{a}_{0} \bar{a}_{i} .
\end{aligned}
$$

Furthermore

$$
\begin{aligned}
v & =\left(x+a_{i}\right)\left(z^{\prime}+a_{k}+a^{*}\right) \leqq\left(y+a_{i}+a_{j}\right)\left(z+\bar{a}_{0} \bar{a}_{i}\right) \\
& =\left(y+a_{i}\right)\left(z+\bar{a}_{0} \bar{a}_{i}\right)+a_{j}=b+a_{j} .
\end{aligned}
$$

Thus $v \leqq b+a_{j} \leqq v+\bar{a}_{0}$, and we have

$$
b+a_{j}=v+\left(b+a_{j}\right) \bar{a}_{0}=v+b \bar{a}_{0}+a_{j}=v+a_{j} .
$$

Since $v$ is contained in $\bar{a}_{j}$, this yields the second equation in (1), and the proof is complete. 
6. Changes in the frame. Throughout this section we assume that $p, q, r$ $=1,2,3$ and $p \neq q \neq r \neq p$. It will be clear from the results in the next section that given two partial frames $a, b \in F$ with $b_{0}, b_{0, p} \in B(a)$, the sets $B(a)$ and $B(b)$ are equal. A direct proof of this fact appears to be difficult, and we shall prove it here only for the special case in which $a_{i}=b_{i}$ for all but one value of $i$.

Lemma 6.1. Suppose $a \in F$. If $d \in B$ and $\bar{a}_{0}=d \dot{+} \bar{a}_{0} \bar{a}_{p}$, then there exists $b \in F$ such that $b_{i}=a_{i}$ whenever $p \neq i<4, b_{p}=d, b^{*}=a^{*}$, and $b_{i, j}=a_{i, j}$ for $i, j$ $=0,1,2,3$ with $i \neq p \neq j$.

Proof. By $1.3, d$ is perspective to $a_{q}$ in $B$. Let $z$ be an axis of such a perspectivity. By the last remark following Definition 1.1 there exists a partial four-frame $b$ of $A^{\prime}$ such that $b_{i}=a_{i}$ whenever $p \neq i<4, b_{p}=d, b^{*}=a^{*}, b_{q, i}$ $=a_{q, i}$ whenever $p \neq i<4$, and $b_{q, p}=x$. Since

$$
b_{0, r}=\left(b_{0, q}+b_{q, r}\right)\left(b_{0}+b_{r}\right)=\left(a_{0, q}+a_{q, r}\right)\left(a_{0}+a_{r}\right)=a_{0, r}
$$

we see that $b$ has all the required properties.

Lemma 6.2. Suppose $a, b \in F$. If $a_{i}=b_{i}$ whenever $p \neq i<4$ and $a^{*}=b^{*}$, then the conditions $b_{0, p} \in B(a)$ and $b_{0, q} \in B(a)$ are equivalent and imply that $B(a)$ $=B(b)$.

Proof. First assume that $b_{p} \leqq \bar{a}_{r}$ and therefore $\bar{a}_{r}=\bar{b}_{r}$. If $b_{0, p} \in B(a)$, then $b_{0, p} \in B_{r}(a)$ by 5.3 , and since $b_{p, q}, b_{0}$ and $b_{q}$ are also members of $B_{r}(a)$ we see that the element $b_{0, q}=\left(b_{0, p}+b_{p, q}\right)\left(b_{0}+b_{q}\right)$ belongs to $B_{r}(a)$, and therefore $b_{0, q} \in B(a)$. For similar reasons $b_{0, q} \in B(a)$ implies that $b_{0, p} \in B(a)$. Assuming now that $b_{0, p}$ and $b_{0, q}$ belong to $B(a)$ and therefore that $b_{0, p} \in B_{r}(a)$ and $b_{0, q} \in B_{p}(a)$, we infer by 1.2 that the elements $b_{0}$ and $b_{0, p}$ together with the set $B \bar{a}_{r}=B \bar{b}_{r}$ generate both $B_{r}(a)$ and $B_{r}(b)$, so that $B_{r}(a)=B_{r}(b)$. Similarly $b_{0}$ and $b_{0, q}$ together with the set $B \bar{a}_{p}=B \bar{b}_{p}$ generate $B_{p}(a)$ and also $B_{p}(b)$, so that $B_{p}(a)=B_{p}(b)$.

In order to prove that $B(a)=B(b)$ it suffices to show that if $c \in A^{\prime}$ and $c u=0$, then the conditions $c \in B(a)$ and $c \in B(b)$ are equivalent. But the element

$$
\left(c+a_{r}\right) \bar{a}_{r}=\left(c+b_{r}\right) b_{r}
$$

belongs to $B_{r}(a)$ if and only if it belongs to $B_{r}(b)$, and the element

$$
\left(c+\bar{a}_{0} \bar{a}_{r}\right)\left(a_{0}+a_{r}\right)=\left(c+b_{0} \bar{b}_{r}\right)\left(b_{0}+b_{r}\right)
$$

belongs to $B_{p}(a)$ if and only if it belongs to $B_{p}(b)$. Using 5.4 we therefore see that $c \in B(a)$ if and only if $c \in B(b)$, as was to be shown.

Considering now the general case, let

$$
x=\left(b_{p}+a_{q}\right) \bar{a}_{q} .
$$

Then $x \in B$ and $\bar{a}_{0}=x+\bar{a}_{0} a_{p}$. By 6.1 there therefore exists $c \in F$ such that 
$c_{i}=a_{i}$ whenever $p \neq i<4, c_{p}=x, c^{*}=a^{*}$, and $c_{i, j}=a_{i, j}$ for $i, j=0,1,2,3$ with $i \neq p \neq j$. Since $c_{0, r}=a_{0, r} \in B(a)$ and $c_{p} \leqq \bar{a}_{q}$, we infer by the special case already considered that $B(a)=B(c)$. Furthermore $b_{p} \leqq x+a_{q}=c_{p}+c_{q} \leqq \bar{c}_{r}$, and using the special case again we infer that the conditions $b_{0, p} \in B(c)$ and $b_{0, q} \in B(c)$ are equivalent and imply that $B(b)=B(c)$. This completes the proof.

Corollary 6.3. Suppose $a \in F$. If $c \in B$ and $c \bar{a}_{p}=0$, then there exists $b \in F$ such that $B(a)=B(b), b_{i}=a_{i}$ whenever $p \neq i<4, c \leqq b_{p}$, and $b^{*}=a^{*}$.

Proof. There exists $d \in B$ such that $\bar{a}_{0}=d+\bar{a}_{0} \bar{a}_{p}$ and $c \leqq d$. Choosing $b$ according to 6.1 we use 6.2 to infer that $B(a)=B(b)$.

Lemma 6.4. Suppose $a \in F$. If $d \in B_{p}(a)$ and $\bar{a}_{p}=d \dot{+} \bar{a}_{0} \bar{a}_{p}$, then there exists $b \in F$ such that $b_{0}=d, b_{i}=a_{i}$ for $i=1,2,3, b_{i, j}=a_{i, j}$ for $i, j=1,2,3, b^{*}=a^{*}$, and $b_{0, q} \in B_{p}(a)$.

Proof. By $1.3 d$ is perspective to $a_{q}$ in $B_{p}(a)$. If $x$ is the axis of such a perspectivity, then we infer by the last remark following Definition 1.1 that there exists a partial four-frame $b$ of $a^{\prime}$ such that $b_{0}=d, b_{i}=a_{i}$ for $i=1,2,3$, $b_{0, q}=x, b_{i, q}=a_{i, q}$ for $i=1,2,3$, and $b^{*}=a^{*}$. Since

$$
b_{p, r}=\left(b_{p, q}+b_{q, r}\right)\left(b_{p}+b_{r}\right)=\left(a_{p, q}+a_{q, r}\right)\left(a_{p}+a_{r}\right)=a_{p, r}
$$

we see that $b \in F$, and that $b$ has the required properties.

Lemma 6.5. Suppose $a, b \in F$. If $a_{i}=b_{i}$ for $i=1,2,3, a^{*}=b^{*}$, and $b_{0}, b_{0, p}$ $\in B(a)$, then $B(a)=B(b)$.

Proof. First assume that $b_{0} \leqq \bar{a}_{q}$. Then

$$
b_{0}+\bar{a}_{0} \bar{a}_{q}=\bar{a}_{q}
$$

and, by $5.3, b_{0}, b_{0, p} \in B_{q}(a)$. We therefore infer with the aid of 1.2 that $B_{q}(a)$ $=B_{q}(b)$.

The perspectivity

$$
x \rightarrow \phi(x)=\left(x+a_{p}\right) \bar{a}_{p}
$$

maps $A^{\prime} \bar{b}_{p}$ isomorphically onto $A^{\prime} \bar{a}_{p}$, and maps each member of $A^{\prime} \bar{a}_{p} \bar{b}_{p}$ onto itself. In particular, each $x \in A^{\prime} \bar{a}_{0} \bar{a}_{p}$ is mapped onto itself. Furthermore, if $x \in B_{q}(a)$ and $x \leqq b_{p}$, then

$$
\phi(x)=\left(x+a_{p}\right) \bar{a}_{p} \bar{a}_{q},
$$

and therefore $\phi(x) \in B_{q}(a)$. In particular $\phi\left(b_{0}\right)$ and $\phi\left(b_{0, r}\right)$ must therefore belong to $B_{q}(a)$, and since they are contained in $\bar{a}_{p}$ it follows that they belong to $B_{p}(a)$. Since, by $1.2, B_{p}(b)$ is generated by the elements $b_{0}$ and $b_{0, p}$ together with the set $B \bar{b}_{p}=B \bar{a}_{p}$ it follows that $\phi$ maps $B_{p}(b)$ into $B_{p}(a)$. Similarly the inverse mapping 


$$
y \rightarrow \psi(y)=\left(y+b_{p}\right) \bar{b}_{p}
$$

carries $B_{p}(a)$ into $B_{p}(b)$, and we conclude that $\phi$ must actually map $B_{p}(b)$ onto $B_{p}(a)$.

Given any $c \in A^{\prime}$ with $c u=0$, the element

$$
x=\left(c+a_{q}\right) \bar{a}_{q}=\left(c+b_{q}\right) b_{q}
$$

is a member $B_{q}(a)$ if and only if it is a member of $B_{q}(b)$. Letting

$$
y=\left(c+b_{p}\right) b_{p}
$$

we have

$$
\phi(y)=\left(c+a_{p}\right) \bar{a}_{p},
$$

and we have shown that $y \in B_{p}(b)$ if and only if $\phi(y) \in B_{p}(a)$. Consequently, by 5.1, $c \in B(a)$ if and only if $c \in B(b)$. This completes the proof for the special case.

We now drop the hypothesis that $b_{0} \leqq \bar{a}_{q}$. Letting

$$
d=\left(b_{0}+a_{q}\right) \bar{a}_{q}
$$

we have $d \in B_{q}(a)$ by 5.1 , and $\bar{a}_{q}=d \dot{+} \bar{a}_{0} \bar{a}_{q}$. By 6.4 there exists $c \in F$ such that $c_{i}=a_{i}$ for $i=1,2,3, c_{0}=d, c^{*}=a^{*}$, and $c_{0, p} \in B_{q}(a)$. By the first part of the proof we therefore have $B(a)=B(c)$. Hence $b_{0}, b_{0, p} \in B(c)$ and $b_{0} \leqq c_{0}+c_{q} \leqq \bar{c}_{r}$. Using the first part of the proof again we finally conclude that $B(c)=B(b)$.

LemmA 6.6. If $a \in F, x \in B(a)$ and $x u=0$, then there exists $b \in F$ such that $B(a)=B(b), x \leqq b_{0}, b_{i}=a_{i}$ for $i=1,2,3$, and $b^{*}=a^{*}$.

Proof. First assume that $x \leqq \bar{a}_{p}$. Then $x \in B_{p}(a)$ by 5.3 , and we can therefore find $d \in B_{p}(a)$ such that

$$
\bar{a}_{p}=\bar{a}_{0} \bar{a}_{p}+d \text { and } x \leqq d .
$$

By 6.4 there exists $b \in F$ such that $b_{0}=d, b_{i}=a_{i}$ for $i=1,2,3, b^{*}=a^{*}$, and $b_{0, q} \in B_{p}(a)$. Consequently $B(a)=B(b)$ by 6.5 .

Considering now the general case, let

$$
x^{\prime}=\left(x+a_{p}\right) \bar{a}_{p} .
$$

By 5.1 and 5.3, $x^{\prime} \in B(a)$. Since $x^{\prime} \leqq \bar{a}_{p}$ and

$$
x^{\prime} u=\left(x+a_{p}\right) \bar{a}_{0} \bar{a}_{p}=\left(x \bar{a}_{0}+a_{p}\right) \bar{a}_{p}=a_{p} \bar{a}_{p}=0,
$$

it follows by the special case already considered that there exists $c \in F$ such that $B(a)=B(c), x^{\prime} \leqq c_{0}, c_{i}=a_{i}$ for $i=1,2,3$ and $c^{*}=a^{*}$. Since $x \leqq x^{\prime}+a_{p}$ $\leqq c_{0}+c_{p}$, we apply the special case a second time to obtain $b \in F$ with the required properties.

7. The closure properties of $B(a)$. Throughout this section we shall assume that $p, q, r=1,2,3$ and $p \neq q \neq r \neq p$. 
Lemma 7.1. Suppose $a \in F$. If $x \in B_{p}(a)$ and $x\left(a_{0}+a_{q}\right)=0$, then the set

$$
C=\left\{y \mid x+a_{0}+a_{p} \geqq y \in B(a)\right\}
$$

is a complemented sublattice of $A^{\prime}$.

Proof. The element $z=\left(x+a_{0}\right) \bar{a}_{0}=\left(x+a_{0}\right) \bar{a}_{0} \bar{a}_{p}$ belongs to $B_{p}(a)$, and therefore $z \in B$ by 4.8 . Hence there exists $d \in B$ such that $\bar{a}_{0} \bar{a}_{p}=d \dot{+} a_{q}$ and $z \leqq d$. Letting

$$
v=\left(a_{r}+a_{q}\right) d, \quad w=\left(a^{*}+a_{q}\right) d
$$

we have $a_{r} \dot{+} a_{q}=v \dot{+} a_{q}$ and $a^{*} \dot{+} a_{q}=w \dot{+} a_{q}$. Consequently there exists a partial four-frame $b$ of $A^{\prime}$ such that $b_{i}=a_{i}$ and $b_{i, j}=a_{i, j}$ for $i, j=0,1,2,3$, and $b^{*}=w$. Clearly $b \in F$, and it is easy to check that $B(a)=B(b)$. In fact, using 4.3 we see that $a^{(0, p)}(x)=b^{(0, p)}(x)$ and $a^{(0, r)}(x)=b^{(0, r)}(x)$, whenever $x \in B$. Hence $B_{p}(a)=B_{p}(b)$ and $B_{r}(a)=B_{r}(b)$. Since $a_{p}=b_{p}$ and $a_{r}=b_{r}$, the conclusion follows with the aid of 5.1. Since $b_{0}=v+b_{0} b_{r}$, it follows from 6.1 that there exists $c \in F$ such that $c_{i}=b_{i}$ whenever $r \neq i<4, c_{r}=v, c^{*}=b^{*}$ and $c_{0, q} \in B_{p}(b)$. Consequently $B(b)=B(c)$ by 6.2 . Thus

$$
C=\left\{y \mid x+c_{0}+c_{p} \geqq y \in B(c)\right\} .
$$

Since $x \leqq \bar{c}_{q}$ and $x \in B(c)$, we use 5.3 to conclude that $x+c_{0}+c_{p} \in B_{q}(c)$ and that $C$ is the principal ideal generated by $x+c_{0}+c_{p}$ in the complemented sublattice $B_{q}(c)$ of $A^{\prime}$.

Lemma 7.2. If $a \in F$ and $c, d \in B(a)$, then $c+d \in B(a)$.

Proof. It is a consequence of 3.3 (viii) that a member $b$ of $A^{\prime}$ belongs to $B(a)$ if and only if

$$
b=b^{\prime}+x
$$

where $b^{\prime} \in B(a), b^{\prime} u=0$ and $x \in B$; we take for $b^{\prime}$ the element $\left(y+a_{i}\right)\left(z+a_{j}\right)$ in 3.3 (viii). From this it readily follows that if $b \in B(a)$ and $c \in B$, then $b+c \in B(a)$. Using this observation we easily reduce the lemma to the special case in which $c u=d u=0$. In fact, assuming that the conclusion has been established for this special case, we have in the general case

$$
c=c_{0}+c_{1}, \quad d=d_{0}+d_{1}
$$

where $c_{0}, d_{0} \in B(a), c_{0} u=d_{0} u=0$ and $c_{1}, d_{1} \in B$. Then $c_{0}+d_{0} \in B(a)$ and $c_{1}+d_{1}$ $\in B$, so that

$$
c+d=\left(c_{0}+d_{0}\right)+\left(c_{1}+d_{1}\right) \in B(a) .
$$

We shall henceforth assume that $c u=d u=0$, and in view of 6.6 we may further assume that $d \leqq a_{0}$. Let

$$
x=\left(c+\bar{a}_{0} \bar{a}_{p}\right)\left(a_{0}+a_{p}\right), \quad y=\left(c+a_{p}\right) \bar{a}_{p} .
$$


Then $x \in B_{q}(a)$ and $y \in B_{p}(a)$ by 5.4 , and therefore

$$
y=y_{0}+y_{1} \text { where } y_{0}=y\left(a_{0}+a_{q}\right) \text { and } y_{1} \in B_{p}(a) .
$$

Let

$$
c_{i}=\left(y_{i}+a_{p}\right)\left(x+\bar{a}_{0} \bar{a}_{p}\right) \quad \text { for } i=0,1 \text {. }
$$

We have

$$
\begin{aligned}
c_{0}+c_{1} & =\left(y_{0}+a_{p}\right)\left(x+\bar{a}_{0} \bar{a}_{p}\right)+\left(y_{1}+a_{p}\right)\left(x+\bar{a}_{0} \bar{a}_{p}\right) \\
& =\left[\left(y_{0}+a_{p}\right)\left(x+\bar{a}_{0} \bar{a}_{p}\right)+y_{1}+a_{p}\right]\left(x+\bar{a}_{0} \bar{a}_{p}\right) \\
& =\left[\left(y_{0}+a_{p}\right)\left(x+\bar{a}_{0}\right)+y_{1}\right]\left(x+\bar{a}_{0} \bar{a}_{p}\right) \\
& =\left(y_{0}+y_{1}+a_{p}\right)\left(x+\bar{a}_{0}\right)\left(x+\bar{a}_{0} \bar{a}_{p}\right) \\
& =\left(y+a_{p}\right)\left(x+\bar{a}_{0} \bar{a}_{p}\right),
\end{aligned}
$$

and therefore, by 5.4, $c=c_{0}+c_{1}$.

Since $y_{0} \in B_{p}(a)$ and $y_{0} \leqq \bar{a}_{r}$, we have $y_{0} \in B_{r}(a)$ by 4.8 . Since the elements $a_{p}, x, a_{q}, a^{*}$ also belong to $B_{r}(a)$, it follows that

$$
c_{0}=\left(y_{0}+a_{p}\right)\left(x+a_{q}+a^{*}\right),
$$

and that $c_{0} \in B_{r}(a)$. Consequently $c_{0}+d \in B_{r}(a)$ and

$$
c_{0}+d=d^{\prime}+z \text { where } z=\left(c_{0}+d\right) u \text { and } d \leqq d^{\prime} \in B_{r}(a) \text {. }
$$

Using 6.6 we can therefore find $b \in F$ such that $B(a)=B(b), d^{\prime} \leqq b_{0} \leqq a_{0}+a_{p}$ $+a_{q}, b_{i}=a_{i}$ for $i=1,2,3$ and $b^{*}=a^{*}$.

We next show that $c_{1} \in B(a)$. Since $y_{1} \in B_{p}(a)$ and $y_{1}\left(a_{0}+a_{q}\right)=0$, it follows from 7.1 that the set

$$
C=\left\{v \mid y_{1}+a_{0}+a_{p} \geqq v \in B(a)\right\}
$$

is a sublattice of $A^{\prime}$. The elements $y_{1}, a_{p}, x$, and $\left(a_{0}+y_{1}\right) \bar{a}_{0} \bar{a}_{p}$ belong to this lattice, and

$$
\begin{aligned}
c_{1} & =\left(y+a_{p}\right)\left(x+\bar{a}_{0} \bar{a}_{p}\right)=\left(y_{1}+a_{p}\right)\left(y_{1}+a_{0}+a_{p}\right)\left(x+\bar{a}_{0} \bar{a}_{p}\right) \\
& =\left(y_{1}+a_{p}\right)\left[x+\left(y_{1}+a_{0}+a_{p}\right) \bar{a}_{0} \bar{a}_{p}\right]=\left(y_{1}+a_{p}\right)\left[x+\left(y_{1}+a_{0}\right) \bar{a}_{0} \bar{a}_{p}\right] .
\end{aligned}
$$

The last equation follows from the fact that $y_{1}, a_{0}$ and $\bar{a}_{0} \bar{a}_{p}$ are contained in $\bar{a}_{p}$. We therefore have $c_{1} \in C$, as was to be shown.

Clearly $\left(y_{1}, a_{0}, a_{p}, a_{q}\right) \perp$, whence it follows that $\left(y_{1}, b_{0}, b_{p}, b_{q}\right) \perp$. Since $y_{1} \in B(b)$ and $y_{1} u=0$, the element

$$
y_{1}^{\prime}=\left(y_{1}+b_{p}\right) b_{p}
$$

belongs to $B_{p}(b)$ by 5.1 . Furthermore,

$$
y_{1}^{\prime}\left(b_{0}+b_{p}+b_{q}\right)=\left(y_{1}+b_{p}\right)\left(b_{0}+b_{q}\right)=0,
$$

and we infer by 7.1 that the set 


$$
D=\left\{v \mid y_{1}+b_{0}+b_{p} \geqq v \in B(a)\right\}=\left\{v \mid y_{1}^{\prime}+b_{0}+b_{p} \geqq v \in B(b)\right\}
$$

is a sublattice of $A^{\prime}$. Since $d^{\prime}, c_{1} \in D$, it follows that $d^{\prime}+c_{1} \in D$, hence $d^{\prime}+c_{1}$ $\in B(a)$, hence $c+d=d^{\prime}+c_{1}+z \in B(a)$. This completes the proof.

Lemma 7.3. If $a \in F$ and $c, d \in B(a)$, then $c d \in B(a)$.

Proof. There exist $c_{0}, d_{0} \in B(a)$ and $c_{1}, d_{1} \in B$ such that

$$
c=c_{0}+c_{1}, \quad d=d_{0}+d_{1}, \quad c_{0} u=d_{0} u=0 .
$$

First observe that the conclusion holds in case $d \in B$, for in this case $c d$ $=c_{1} d \in B$. In particular we have $c u \in B$ for all $c \in B(a)$.

Returning now to the general case, we see by 6.6 that we may assume that $c_{0} \leqq a_{0}$. By 7.2 and the observation just made, the elements

$$
c_{2}=c_{1}\left(c_{0}+d_{0}+d_{1}\right), \quad d_{2}=d_{1}\left(c_{0}+c_{1}+d_{0}\right)
$$

belong to $B$ and therefore the elements $c_{0}+c_{2}$ and $d_{0}+d_{2}$ belong to $B(a)$. Furthermore,

$$
\begin{aligned}
& c_{0}+c_{2}=c_{0}+c_{1}\left(c_{0}+d_{0}+d_{1}\right)=\left(c_{0}+c_{1}\right)\left(c_{0}+d_{0}+d_{1}\right) \\
& d_{0}+d_{2}=d_{0}+d_{1}\left(c_{0}+c_{2}+d_{0}\right)=\left(d_{0}+d_{1}\right)\left(c_{0}+c_{1}+d_{0}\right)
\end{aligned}
$$

so that

$$
\left(c_{0}+c_{2}\right)\left(d_{0}+d_{2}\right)=c d .
$$

It is also easy to check that

$$
c_{0}+d_{0}+c_{2}=c_{0}+d_{0}+d_{2},
$$

whence it follows that the mapping

$$
x \rightarrow \phi(x)=\left(x+c_{0}\right)\left(d_{0}+d_{2}\right)
$$

is an isomorphism of the quotient $c_{2} / 0$ of $A^{\prime}$ into the quotient $\left(d_{0}+d_{2}\right) / c_{0}\left(d_{0}+d_{2}\right)$. In fact, the mapping $x \rightarrow x+c_{0}$ is an isomorphism of $c_{2} / 0$ onto $c_{0}+c_{2} / c_{0}$ and therefore into $\left(c_{0}+d_{0}+d_{2}\right) / c_{0}$, while the mapping $y \rightarrow y\left(d_{0}+d_{2}\right)$ is an isomorphism of this last quotient onto $\left(d_{0}+d_{2}\right) / c_{0}\left(d_{0}+d_{2}\right)$. Now $c_{2}$ can be written in the form $c_{2}=x+y$ where $x, y \in B, x \leqq \bar{a}_{p}$ and $y \bar{a}_{p}=0$, and we have

$$
c d=\phi\left(c_{2}\right)=\phi(x)+\phi(y) .
$$

Because of 7.2 it is therefore sufficient to show that $\phi(x)$ and $\phi(y)$ belong to $B(a)$, i.e., we need only consider the special cases $c_{1} \leqq \bar{a}_{p}$ and $c_{1} \bar{a}_{p}=0$. It follows from 6.3 that in the second case we may assume that $c_{1} \leqq a_{p}$. Similarly we may assume that either $d_{2} \leqq \bar{a}_{p}$ or $d_{2} \bar{a}_{p}=0$. Accordingly our problem reduces to four special cases.

CASE 1. $c_{2} \leqq \bar{a}_{p}$ and $d_{2} \leqq \bar{a}_{p}$. 
In this case $c_{0}+c_{2} \leqq \bar{a}_{p}$ and hence

$$
c d=\left(c_{0}+c_{2}\right)\left(d_{0} \bar{a}_{p}+d_{2}\right),
$$

and since $B_{p}(a)$ is a sublattice of $A^{\prime}$ and a subset of $B(a)$, it is sufficient to show that $d_{0} \bar{a}_{p} \in B_{p}(a)$. By 5.4 the elements

$$
x=\left(d_{0}+a_{p}\right) \bar{a}_{p}, \quad y=\left(d_{0}+\bar{a}_{0} \bar{a}_{p}\right)\left(a_{0}+a_{p}\right)
$$

are members of $B_{p}(a)$ and of $B_{q}(a)$, respectively. Therefore $y a_{0} \in B_{q}(a)$, and it follows by 4.8 that $y a_{0} \in B_{p}(a)$. Consequently the element

$$
z=x\left(y a_{0}+\bar{a}_{0} \bar{a}_{p}\right)
$$

belongs to $B_{p}(a)$. Furthermore,

$$
\begin{aligned}
z & =\left(d_{0}+a_{p}\right)\left[\left(d_{0}+\bar{a}_{0} \bar{a}_{p}\right) a_{0}+\bar{a}_{0} \bar{a}_{p}\right] \\
& =\left(d_{0}+a_{p}\right)\left(d_{0}+\bar{a}_{0} \bar{a}_{p}\right) \bar{a}_{p}=\left(d_{0}+a_{p}\right)\left(d_{0} \bar{a}_{p}+\bar{a}_{0} \bar{a}_{p}\right) \\
& =d_{0} \bar{a}_{p}+\left(d_{0}+a_{p}\right) \bar{a}_{0} \bar{a}_{p}=d_{0} \bar{a}_{p} .
\end{aligned}
$$

The last equality holds because $\left(\bar{a}_{0} \bar{a}_{p}, a_{p}, d_{0}\right) \perp$. Consequently $d_{0} \bar{a}_{p} \in B_{p}(a)$, as was to be shown.

CASE 2. $c_{2} \leqq a_{p}$ and $d_{2} \leqq \bar{a}_{p}$.

We have

$$
c d=\left(c_{0}+c_{2}\right)\left(d_{0}+d_{2}\right)\left(a_{0}+a_{p}\right)
$$

and since $c_{0}+c_{2} \in B_{q}(a)$, it is sufficient to show that $\left(d_{0}+d_{2}\right)\left(a_{0}+a_{p}\right)$ is a member of $B_{q}(a)$. Let

$$
\begin{array}{ll}
x=\left(d_{0}+a_{p}\right) \bar{a}_{p}, & y=\left(d_{0}+\bar{a}_{0} \bar{a}_{p}\right)\left(a_{0}+a_{p}\right), \\
z=\left(x+d_{2}\right) a_{0}, & v=\left(z+a_{p}\right) y .
\end{array}
$$

By 5.4, $x \in B_{p}(a)$ and $y \in B_{q}(a)$. We infer that $z \in B_{p}(a)$, hence $z \in B_{q}(a)$ by 4.8, and hence $v \in B_{q}(a)$. Furthermore

$$
\begin{aligned}
z+a_{p} & =\left[\left(d_{0}+a_{p}\right) \bar{a}_{p}+d_{2}\right] a_{0}+a_{p}=\left(d_{0}+d_{2}+a_{p}\right) \bar{a}_{p} a_{0}+a_{p} \\
& =\left(d_{0}+d_{2}+a_{p}\right) a_{0}+a_{p}=\left(d_{0}+d_{2}+a_{p}\right)\left(a_{0}+a_{p}\right) \\
& =a_{p}+\left(d_{0}+d_{2}\right)\left(a_{0}+a_{p}\right)
\end{aligned}
$$

and consequently

$$
\begin{aligned}
v & =\left[a_{p}+\left(d_{0}+d_{2}\right)\left(a_{0}+a_{p}\right)\right]\left(d_{0}+\bar{a}_{0} \bar{a}_{p}\right) \\
& =a_{p}\left(d_{0}+\bar{a}_{0} \bar{a}_{p}\right)+\left(d_{0}+d_{2}\right)\left(a_{0}+a_{p}\right)=\left(d_{0}+d_{2}\right)\left(a_{0}+a_{p}\right) .
\end{aligned}
$$

Thus $\left(d_{0}+d_{2}\right)\left(a_{0}+a_{p}\right) \in B_{q}(a)$, as was to be shown.

CASE 3. $c_{2} \leqq \bar{a}_{p}$ and $d_{2} \bar{a}_{p}=0$.

In view of 6.3 we may assume that $d_{2} \leqq a_{p}$. By 6.6 there exists $b \in F$ such that $B(a)=B(b), d_{0} \leqq b_{0}, b_{i}=a_{i}$ for $i=1,2,3$, and $b^{*}=a^{*}$. This case therefore reduces to the preceding one, with $c_{i}$ and $d_{i}$ interchanged. 
CASE 4. $c_{2} \leqq a_{p}$ and $d_{2} \bar{a}_{p}=0$.

An argument similar to the one that reduced the original problem to the four special cases under consideration also shows that in the present case we may assume that either $d_{2} \leqq \bar{a}_{q}$ or else $d_{2} \leqq a_{q}$. In the two subcases that result, the conclusion follows from the first and the third case, respectively.

The proof is now complete.

Theorem 7.4. For any $a \in F, B(a)$ is a complemented sublattice of $A^{\prime}$, and $B$ is an ideal of $B(a)$.

Proof. By 7.2 and 7.3, $B(a)$ is a sublattice of $A^{\prime}$. If $c \in B(a)$, then $c u \in B$ and there exists $x \in B$ such that $u=c u+x$. The element $a_{0}(c+x)$ belongs to the complemented sublattice $B_{p}(a)$ of $B(a)$, hence there exists $y \in B_{p}(a)$ such that $a_{0}=a_{0}(c+x)+y$, and it is easy to check that $u^{\prime}=c \dot{+}(x+y)$. Thus $B(a)$ is complemented. Finally, by $5.3, B_{i}(a)$ is an ideal of $B(a)$, and hence in particular $B=B_{0}(a)$ is an ideal of $B(a)$.

The four statements from which Theorem 3.2 was shown to follow have now been established, and the proof of Theorem 3.2 is therefore complete.

8. The existence of a representation. The following definition was stated informally in the introduction.

Definition 8.1. A lattice $B$ is said to be COORDinatizable if and only if $B \cong L(R)$ for some regular ring $R$.

LEMMA 8.2. Every principal ideal in a coordinatizable lattice is coordinatizable.

Proof. Consider a principal ideal $C=B b$ of a coordinatizable lattice $B$. By hypothesis there exists an isomorphism $f$ of $B$ onto the lattice $L(R)$ of all principal left ideals of a regular ring $R$. Since $f(b)$ is a principal left ideal of $R$, we have $f(b)=R \epsilon$ for some idempotent element $\epsilon \in R$. The subring $R^{\prime}=\epsilon R \epsilon$ is regular, and we shall show that $C$ is mapped isomorphically onto $L\left(R^{\prime}\right)$ by the correspondence

$$
x \rightarrow g(x)=R^{\prime} \cap f(x) .
$$

First observe that if $\alpha \in R^{\prime}$ is idempotent, then $R^{\prime} \cap R \alpha=R^{\prime} \alpha$. In fact, if $\beta \in R^{\prime} \cap R \alpha$, then $\beta=\epsilon \beta=\beta \epsilon=\beta \alpha$, and hence $\beta=\epsilon \beta \epsilon \alpha \in R^{\prime} \alpha$. Consequently $R^{\prime} \cap R \alpha \subseteq R^{\prime} \alpha$. The opposite conclusion is obvious.

If $x \in C$, then $x \leqq b$ and hence $f(x) \subseteq f(b)$. Therefore $f(x)=R \beta$ for some $\beta \in R \epsilon$. We have $\beta=\beta \gamma \beta$ for some $\gamma \in R$, and hence $\beta=\beta \alpha$ where $\alpha=\epsilon \gamma \beta$. Clearly $\alpha \in R^{\prime}, \alpha$ is idempotent, and $R \beta=R \alpha$. Consequently $g(x)=R^{\prime} \alpha$ $\in L\left(R^{\prime}\right)$. Conversely, every member of $L\left(R^{\prime}\right)$ is of the form $R^{\prime} \alpha$ for some idempotent $\alpha \in R^{\prime}$. Therefore $R \alpha=f(x)$ for some $x \in B$, and since $\alpha \in R \in$ we have $R \alpha \subseteq R \epsilon$, hence $x \leqq b$, and we conclude that $g(x)=R^{\prime} \cap R \alpha=R^{\prime} \alpha$. Thus $g$ maps $C$ onto $L\left(R^{\prime}\right)$.

Suppose $x, y \in C$ and $g(x) \subseteq g(y)$. Then there exist idempotent elements 
$\alpha, \beta \in R^{\prime}$ such that $f(x)=R \alpha$ and $f(y)=R \beta$, and hence $g(x)=R^{\prime} \alpha$ and $g(y)$ $=R^{\prime} \beta$. Since $R^{\prime} \alpha \subseteq R^{\prime} \beta$, we have $\alpha \in R^{\prime} \beta \subseteq R \beta$, hence $R \alpha \subseteq R \beta, f(x) \subseteq f(y)$, $x \leqq y$. We therefore see that $g$ is one-to-one, and being order-preserving it must therefore be an isomorphism. Consequently $C$ is coordinatizable.

THEOREм 8.3. Every complemented Arguesian lattice which possesses a large partial three-frame is coordinatizable.

Proof. Suppose $B$ is a complemented Arguesian lattice with unit element $u$, and assume that $a$ is a large partial three-frame of $B$. Then

$$
a^{*}=\sum_{i<n} x_{i}
$$

where each of the elements $x_{i}$ is perspective to a part of $a_{0}$. Applying Theorem $2.1 n$ times we embed $B$ as an ideal in a complemented Arguesian lattice $B^{\prime}$ in such a way that

$$
B^{\prime}=B+B b_{0}+B b_{1}+\cdots+B b_{n-1}
$$

where the elements $b_{0}, b_{1}, \cdots, b_{n-1} \in B^{\prime}$ are perspective to $a_{0}$. Since $\left(a_{0}, b_{i}, x_{i}\right) \perp$, it follows by 1.3 that $x_{i}$ is perspective to an element $y_{i} \leqq b_{i}$. Consequently $u$ is easily seen to be perspective to the element

$$
v=a_{0}+a_{1}+a_{2}+y_{0}+y_{1} \dot{+} \cdots+y_{n-1}
$$

and $B$ is therefore isomorphic to the ideal $B^{\prime} v$. Let

$$
w=a_{0}+a_{1}+a_{2}+b_{0}+b_{1}+\cdots+b_{n-1} \text {. }
$$

Then $B^{\prime} w$ possesses a global $(n+3)$-frame, and is therefore coordinatizable, whence it follows by 8.2 that $B^{\prime} v$ is also coordinatizable. Thus $B$ is coordinatizable.

In the above theorem we did of course make essential use of von Neumann's coordinate theorem for a complemented modular lattice $B$ which possesses an $n$-frame $a$ with $n \geqq 4$. The proof of von Neumann's theorem proceeds in two stages. First the auxiliary ring $R$ is constructed and it is shown that $B a_{0}$ is isomorphic to $L(R)$, and then it is shown that $B$ is isomorphic to $L\left(R_{n}\right)$ where $R_{n}$ is the ring of all $n \times n$ matrices over $R$. Since all the known proofs of this theorem are quite long, it is of some interest to observe that actually only the first part of this result is needed here, for applying Theorem $3.14 n+12$ times we can embed our lattice $B$ of Theorem 8.3 as an ideal in a complemented modular lattice $B^{\prime \prime}$ which possesses a four-frame $b$ such that $u \leqq b_{0}$. Consequently, if $B^{\prime \prime} b_{0}$ is coordinatizable, then so is $B$ by 8.2.

COROLlaRy 8.4. Every complemented modular lattice which possesses a large partial four-frame is coordinatizable. 
Proof. By 2.1, such a lattice is necessarily Arguesian, and the conclusion therefore follows from 8.3.

COROLlaRy 8.5. Every simple complemented modular lattice of dimension four or more is coordinatizable.

Proof. By 1.7 and 8.4.

9. The isomorphism theorem. We shall prove that under the hypothesis of 8.3 the ring $R$ in the conclusion of that theorem is uniquely determined up to isomorphism. The basic ideas of the proof are the same as in von Neumann's proof for the case when the lattice possesses a global $n$-frame, although in the general case $R$ will not be isomorphic to a full matrix ring over the auxiliary ring, but rather to a subring of this ring. The next lemma is actually known from the literature. (Cf. [8, p. 149].)

LemMa 9.1. Suppose $\epsilon_{0}$ and $\epsilon_{1}$ are idempotent elements of a regular ring $R$ and $\epsilon_{0} \epsilon_{1}=\epsilon_{1} \epsilon_{0}=0$. If the elements $R \epsilon_{0}$ and $R \epsilon_{1}$ of $L(R)$ are perspective with $c \in L(R)$ as an axis of perspectivity, then there exist unique elements $\alpha$ and $\beta$ of $R$ such that

$$
\begin{gathered}
\epsilon_{0} \alpha=\alpha \epsilon_{1}=\alpha, \quad \epsilon_{1} \beta=\beta \epsilon_{0}=\beta, \quad \epsilon_{0}=\alpha \beta, \quad \epsilon_{1}=\beta \alpha, \\
c=R\left(\epsilon_{0}-\alpha\right)=R\left(\epsilon_{1}-\beta\right) .
\end{gathered}
$$

Proof. We have

(1) $\epsilon_{0}=\alpha+\alpha^{\prime}, \quad \epsilon_{1}=\beta+\beta^{\prime} \quad$ with $\quad \alpha \in R \epsilon_{1}, \quad \beta \in R \epsilon_{0}, \quad \alpha^{\prime}, \beta^{\prime} \in c$.

Therefore $\alpha=\alpha \epsilon_{1}$ and $\beta=\beta \epsilon_{0}$. Also

$$
\left(\epsilon_{0}-1\right) \alpha=\left(1-\epsilon_{0}\right)\left(\epsilon_{0}-\alpha\right)=\left(1-\epsilon_{0}\right) \alpha^{\prime} \in R \epsilon_{1} \cap c=0,
$$

so that $\epsilon_{0} \alpha=\alpha$. Similarly $\epsilon_{1} \beta=\beta$. Since

$$
R \epsilon_{0} \subseteq R \alpha+R \alpha^{\prime} \subseteq R \epsilon_{1}+R \alpha^{\prime},
$$

we have

$$
R \epsilon_{1}+c=R \epsilon_{1}+R \epsilon_{0} \subseteq R \epsilon_{1}+R \alpha^{\prime},
$$

and since $R \alpha^{\prime} \subseteq c$ and $R \epsilon_{1} \cap c=0$, we must have $c=R \alpha^{\prime}=R\left(\epsilon_{0}-\alpha\right)$. Similarly $c=R\left(\epsilon_{1}-\beta\right)$. It follows that

$$
\epsilon_{0}-\alpha=\lambda\left(\epsilon_{1}-\beta\right) \quad \text { for some } \lambda \in R,
$$

and therefore

$$
\begin{gathered}
\alpha=\epsilon_{0}-\lambda\left(\epsilon_{1}-\beta\right), \quad \alpha=\alpha \epsilon_{1}=-\lambda \epsilon_{1}, \\
\alpha \beta=-\lambda \epsilon_{1} \beta=-\lambda \beta=\epsilon_{0}-\alpha-\lambda \epsilon_{1}=\epsilon_{0} .
\end{gathered}
$$

Similarly $\beta \alpha=\epsilon_{1}$. Thus $\alpha$ and $\beta$ have the required properties.

Conversely, if $\alpha$ and $\beta$ satisfy the conditions of the lemma, then the elements $\alpha^{\prime}=\epsilon_{0}-\alpha$. and $\beta^{\prime}=\epsilon_{1}-\beta$ belong to $c$, and (1) holds. But the elements 
$\alpha, \beta, \alpha^{\prime}, \beta^{\prime}$ in (1) are unique, and this proves the uniqueness part of our lemma.

DEFINITION 9.2. By a GENERALIZED SYSTEM OF $n \times n$ MATRIX UNITS over a regular ring $R$ we mean an ordered quadruple $\langle\epsilon, \eta, \alpha, \beta\rangle$ of $n$-termed sequences whose terms $\epsilon_{i}, \eta_{i}, \alpha_{i}, \beta_{i}$ belong to $R$ and satisfy the following conditions:

(i) $\epsilon_{0}, \epsilon_{1}, \cdots, \epsilon_{n-1}$ are idempotent, $\epsilon_{0}+\epsilon_{1}+\cdots+\epsilon_{n-1}=1$, and $\epsilon_{i} \epsilon_{j}=0$ for all $i, j<n$ with $i \neq j$.

(ii) $\eta_{0}=\epsilon_{0}$ and, for $i=1,2, \cdots, n-1, \eta_{i}$ is idempotent and $\eta_{i} \epsilon_{0}=\epsilon_{0} \eta_{i}=\eta_{i}$.

(iii) $\alpha_{0}=\beta_{0}=\epsilon_{0}$ and, for $i=1,2, \cdots, n-1, \eta_{i} \alpha_{i}=\alpha_{i} \epsilon_{i}=\alpha_{i}, \epsilon_{i} \beta_{i}=\beta_{i} \eta_{i}=\beta_{i}$, $\eta_{i}=\alpha_{i} \beta_{i}$ and $\epsilon_{i}=\beta_{i} \alpha_{i}$.

LEMMA 9.3. If $\langle\epsilon, \eta, \alpha, \beta\rangle$ is a generalized system of $n \times n$ matrix units over a regular ring $R$, then $R$ is isomorphic to a subring of the ring of all $n \times n$ matrices over $\epsilon_{0} R \epsilon_{0}$ under the correspondence $f$ such that $f(\gamma)_{i, j}=\alpha_{i} \gamma \beta_{j}$ for all $\gamma \in R$, and for $i, j=0,1, \cdots, n-1$. The image of $R$ consists of all those matrices $m$ for which $\eta_{i} m_{i, j}=m_{i, j} \eta_{j}=m_{i, j}$ for $i, j=0,1, \cdots, n-1$, and the inverse mapping is the function $g$ such that

$$
g(m)=\sum_{i, j<n} \beta_{i} m_{i, j} \alpha_{j} .
$$

Proof. Let $S$ be the set of all $n \times n$ matrices $m$ such that $\eta_{i} m_{i, j}=m_{i, j} \eta_{j}$ $=m_{i, j}$ for $i, j=0,1, \cdots, n-1$. Clearly $f$ maps $R$ into $S$ and $g$ maps $S$ into $R$.

If $\gamma \in R$ and $m=f(\gamma)$, then

$$
g(m)=\sum_{i, j<n} \beta_{i} m_{i, j} \alpha_{j}=\sum_{i, j<n} \beta_{i} \alpha_{i} \gamma \beta_{j} \alpha_{j}=\sum_{i, j<n} \epsilon_{i} \gamma \epsilon_{j}=\gamma .
$$

If $m \in S$ and $\gamma=g(m)$, then for $p, q=0,1, \cdots, n-1$,

$$
f(\gamma)_{p, q}=\alpha_{p} \gamma \beta_{q}=\sum_{i, j<n} \alpha_{p} \beta_{i} m_{i, j} \alpha_{j} \beta_{q}=\eta_{p} m_{p, q} \eta_{q}=m_{p, q}
$$

Here we used the fact that if $i \neq p$, then $\alpha_{p} \beta_{i}=\alpha_{p} \epsilon_{p} \epsilon_{i} \beta_{i}=0$. Thus we see that $f$ maps $R$ in a one-to-one manner onto $S$, and that $g$ is the inverse of $f$.

Suppose $m, m^{\prime} \in S$. Clearly $g\left(m+m^{\prime}\right)=g(m)+g\left(m^{\prime}\right)$. Also,

$$
\begin{gathered}
g\left(m m^{\prime}\right)=\sum_{i, p<n} \beta_{i}\left(\sum_{j<n} m_{i, j} m_{j, p}^{\prime}\right) \alpha_{p}=\sum_{i, j, p<n} \beta_{i} m_{i, j} m_{j, p}^{\prime} \alpha_{p}, \\
g(m) g\left(m^{\prime}\right)=\left(\sum_{i, j<n} \beta_{i} m_{i, j} \alpha_{j}\right)\left(\sum_{p, q<n} \beta_{p} m_{p, q} \alpha_{q}\right) \\
=\sum_{i, j, p, q<n} \beta_{i} m_{i, j} \alpha, \beta_{p} m_{p, q} \alpha_{q} \\
=\sum_{i, j, q<n} \beta_{i} m_{i, j} m_{j, q}^{\prime} \alpha_{q}=g\left(m m^{\prime}\right) .
\end{gathered}
$$

Thus $g$ is an isomorphism, and hence so is $f$. 
Theorem 9.4. Suppose $B$ is a complemented modular lattice and assume that there exists a large partial three-frame $a$ of $B$. If $R$ and $R^{\prime}$ are regular rings such that $L(R) \cong L\left(R^{\prime}\right) \cong B$, then $R \cong R^{\prime}$.

Proof. If $u$ is the unit element of $B$, then

$$
u=\sum_{i<n} b_{i}
$$

where $b_{i}=a_{i}$ for $i=0,1,2$, and each of the elements $b_{i}$ is perspective to some element $x_{i} \leqq b_{0}$. Let $c_{i}$ be the axis of such a perspectivity, and also choose $y_{i} \in B$ with $b_{0}=x_{i}+y_{i}$. In particular, for $i=0,1,2$ we have $x_{i}=b_{0}$ and $y_{i}=0$.

If $f$ is an isomorphism of $B$ onto $L(R)$, then there exist idempotent elements $\epsilon_{0}, \epsilon_{1}, \cdots, \epsilon_{n-1} \in R$ such that

$$
\begin{array}{r}
1=\epsilon_{0}+\epsilon_{1}+\cdots+\epsilon_{n-1}, \epsilon_{i} \epsilon_{j}=0 \text { for } i, j=0,1, \cdots, n-1 \text { with } i \neq j, \\
f\left(b_{i}\right)=R \epsilon_{i} \quad \text { for } i=0,1, \cdots, n-1 .
\end{array}
$$

For each $i<n$ there exists an idempotent $\eta_{i} \in R$ such that

$$
\eta_{i} \epsilon_{0}=\epsilon_{0} \eta_{i}=\eta_{i}, \quad f\left(x_{i}\right)=R \eta_{i}, \quad f\left(y_{i}\right)=R\left(\epsilon_{0}-\eta_{i}\right),
$$

and by 9.1 there exist unique elements $\alpha_{i}, \beta_{i} \in R$ such that

$$
\begin{gathered}
\eta_{i} \alpha_{i}=\alpha_{i} \epsilon_{i}=\alpha_{i}, \quad \epsilon_{i} \beta_{i}=\beta_{i} \eta_{i}=\beta_{i}, \quad \eta_{i}=\alpha_{i} \beta_{i}, \quad \epsilon_{i}=\beta_{i} \alpha_{i}, \\
f\left(c_{i}\right)=R\left(\eta_{i}-\alpha_{i}\right)=R\left(\epsilon_{i}-\beta_{i}\right) .
\end{gathered}
$$

Thus $\langle\epsilon, \eta, \alpha, \beta\rangle$ is a generalized system of matrix units of $R$ and, by 9.3 , $R$ is isomorphic to the ring $S$ consisting of all those $n \times n$ matrices $m$ over $\epsilon_{0} R \epsilon_{0}$ which satisfy the condition

$$
\eta_{i} m_{i, j}=m_{i, j} \eta_{j}=m_{i, j} \quad \text { for } i, j=0,1, \cdots, n-1 .
$$

Similarly, if $f^{\prime}$ is an isomorphism of $B$ onto $L\left(R^{\prime}\right)$, then there exists a generalized system of matrix units for $R^{\prime},\left\langle\epsilon^{\prime}, \eta^{\prime}, \alpha^{\prime}, \beta^{\prime}\right\rangle$, such that

$$
\begin{aligned}
& f^{\prime}\left(b_{i}\right)=R^{\prime} \epsilon_{i}^{\prime}, \quad f^{\prime}\left(x_{i}\right)=R^{\prime} \eta_{i}^{\prime}, \quad f^{\prime}\left(y_{i}\right)=R^{\prime}\left(\epsilon_{i}^{\prime}-\eta_{i}^{\prime}\right), \\
& f^{\prime}\left(c_{i}\right)=R^{\prime}\left(\eta_{i}^{\prime}-\alpha_{i}^{\prime}\right)=R^{\prime}\left(\epsilon_{i}^{\prime}-\beta_{i}^{\prime}\right) .
\end{aligned}
$$

Consequently $R^{\prime}$ is isomorphic to the ring $S^{\prime}$ of all those $n \times n$ matrices $m^{\prime}$ over $\epsilon_{0}^{\prime} R^{\prime} \epsilon_{0}^{\prime}$ which satisfy the condition

$$
\eta_{i}^{\prime} m_{i, j}^{\prime}=m_{i, j}^{\prime} \eta_{j}^{\prime}=m_{i, j}^{\prime} \quad \text { for } i, j=0,1, \cdots, n-1 .
$$

The proof of the theorem will therefore be completed if we show that there exists an isomorphism of $\epsilon_{0} R \epsilon_{0}$ onto $\epsilon_{0}^{\prime} R^{\prime} \epsilon_{0}^{\prime}$ which maps $\eta_{i}$ onto $\eta_{i}^{\prime}$ for all $i<n$.

For each $\gamma \in \epsilon_{0} R \epsilon_{0}$ there exists a unique element $g(\gamma) \in B$ such that $f g(\gamma)$ $=R\left(\gamma-\alpha_{1}\right)$. We shall show that $g$ is a one-to-one mapping of $\epsilon_{0} R \epsilon_{0}$ onto the set 


$$
T=\left\{v \mid v \in B \text { and } b_{0}+b_{1}=b_{0}+v\right\} .
$$

Since $\gamma \in R \epsilon_{0}=f\left(b_{0}\right)$ and $\alpha_{1}=\alpha_{1} \epsilon_{1} \in R \epsilon_{1}=f\left(b_{1}\right)$, we have $g(\gamma) \leqq b_{0}+b_{1}$. Since

$$
\epsilon_{1}=\beta_{1} \alpha_{1}=\beta_{1} \gamma-\beta_{1}\left(\gamma-\alpha_{1}\right) \in R \epsilon_{0}+R\left(\gamma-\alpha_{1}\right)=f\left(b_{0}\right)+f g(\gamma),
$$

we have $b_{1} \leqq b_{0}+g(\gamma)$. If $\delta \in f\left(b_{0}\right) \cap f g(\gamma)=R \epsilon_{0} \cap R\left(\gamma-\alpha_{1}\right)$, then $\delta=\delta \epsilon_{0}$ and $\delta=\lambda\left(\gamma-\alpha_{1}\right)$ for some $\lambda \in R$. Therefore $\delta=\lambda\left(\gamma-\alpha_{1}\right) \epsilon_{0}=\lambda \gamma, \lambda \alpha_{1}=0, \lambda \epsilon_{0}=\lambda \alpha_{1} \beta_{1}$ $=0, \lambda \gamma=\lambda \epsilon_{0} \gamma=0$, and we conclude that $\delta=0$. Thus $b_{0} g(\gamma)=0$. We have therefore shown that $g(\gamma) \in T$ for all $\gamma \in \epsilon_{0} R \epsilon_{0}$.

If $v \in T$, there exist unique elements $\gamma \in R \epsilon_{0}$ and $\delta \in f(v)$ such that $\alpha_{1}=\gamma+\delta$. Since $\alpha_{1}=\epsilon_{0} \alpha_{1}=\epsilon_{0} \gamma+\epsilon_{0} \delta$, we must have $\gamma=\epsilon_{0} \gamma \in \epsilon_{0} R \epsilon_{0}$. Also, the fact that $R \epsilon_{1}=R \alpha_{1} \subseteq R \gamma+R \delta \subseteq R \epsilon_{0}+R \delta$ and $R \delta \subseteq f(v)$ implies that $f(v)=R \delta$ $=R\left(\gamma-\alpha_{1}\right)$, hence $v=g(\gamma)$. Thus $g$ maps $\epsilon_{0} R \epsilon_{0}$ onto $T$.

If $\gamma, \delta \in \epsilon_{0} R \epsilon_{0}$ and $g(\gamma)=g(\delta)$, then $\delta-\alpha_{1}=\lambda\left(\gamma-\alpha_{1}\right)$ for some $\lambda \in R$. Therefore $\delta=\delta \epsilon_{0}=\left(\delta-\alpha_{1}\right) \epsilon_{0}=\lambda\left(\gamma-\alpha_{1}\right) \epsilon_{0}=\lambda \gamma, \lambda \alpha_{1}=\lambda \gamma-\delta+\alpha_{1}=\alpha_{1}, \lambda \epsilon_{0}=\lambda \alpha_{1} \beta_{1}=\alpha_{1} \beta_{1}$ $=\epsilon_{0}, \lambda\left(\gamma-\alpha_{1}\right)=\lambda \epsilon_{0}\left(\gamma-\alpha_{1}\right)=\epsilon_{0}\left(\gamma-\alpha_{1}\right)=\gamma-\alpha_{1}$. Thus we conclude that $\gamma=\delta$.

Having shown that $g$ is a one-to-one mapping of $\epsilon_{0} R \epsilon_{0}$ onto $T$, we can define operations $\oplus$ and $\odot$ of addition and multiplication in $T$ in such a way $T$ becomes a regular ring and $g$ maps $\epsilon_{0} R \epsilon_{0}$ isomorphically onto $T$.

We next express the ring operations in $T$ in terms of the lattice operations in $B$. Let

$$
d=\left(c_{1}+c_{2}\right)\left(b_{1}+b_{2}\right) .
$$

Since $f\left(c_{i}\right)=R\left(\epsilon_{0}-\alpha_{i}\right)$ and $f\left(b_{i}\right)=R \epsilon_{i}$, every element $\xi \in f(d)$ can be expressed in the form

$$
\xi=\lambda_{1}\left(\epsilon_{0}-\alpha_{1}\right)+\lambda_{2}\left(\epsilon_{0}-\alpha_{2}\right)=\mu_{1} \epsilon_{1}+\mu_{2} \epsilon_{2}
$$

for some $\lambda_{1}, \lambda_{2}, \mu_{1}, \mu_{2} \in R$. Therefore $\xi \epsilon_{0}=\lambda_{1} \epsilon_{0}+\lambda_{2} \epsilon_{0}=0, \lambda_{2} \epsilon_{0}=-\lambda_{1} \epsilon_{0}$, $\xi=\lambda_{1}\left(\epsilon_{0}-\alpha_{1}-\epsilon_{0}+\alpha_{2}\right)=-\lambda_{1}\left(\alpha_{1}-\alpha_{2}\right)$. Since clearly $\alpha_{1}-\alpha_{2} \in f(d)$, we thus find that $f(d)=R\left(\alpha_{1}-\alpha_{2}\right)$.

We shall now show that, for all $v, w \in T$,

$$
\begin{aligned}
& v \oplus w=\left[(v+d)\left(b_{0}+b_{2}\right)+\left(w+b_{2}\right)\left(b_{0}+d\right)\right]\left(b_{0}+b_{1}\right) . \\
& v \odot w=\left[\left(v+c_{2}\right)\left(b_{1}+b_{2}\right)+(w+d)\left(b_{0}+b_{2}\right)\right]\left(b_{0}+b_{1}\right) .
\end{aligned}
$$

Let

$$
\begin{array}{ll}
z_{0}=(v+d)\left(b_{0}+b_{2}\right), & z_{1}=\left(w+b_{2}\right)\left(b_{0}+d\right), \\
z_{2}=\left(z_{0}+z_{1}\right)\left(b_{0}+b_{1}\right), & z_{3}=\left(v+c_{2}\right)\left(b_{1}+b_{2}\right), \\
z_{4}=(w+d)\left(b_{0}+b_{2}\right), & z_{5}=\left(z_{3}+z_{4}\right)\left(b_{0}+b_{1}\right) .
\end{array}
$$

There exist $\gamma, \delta \in \epsilon_{0} R \epsilon_{0}$ such that $v=g(\gamma)$ and $w=g(\delta)$. If $\xi \in f\left(z_{0}\right)$, then $\xi=\lambda_{1}\left(\gamma-\alpha_{1}\right)+\lambda_{2}\left(\alpha_{1}-\alpha_{2}\right)=\mu_{1} \epsilon_{0}+\mu_{2} \epsilon_{2}$ for some $\lambda_{1}, \lambda_{2}, \mu_{1}, \mu_{2} \in R$. Therefore $\xi \epsilon_{1}=-\lambda_{1} \alpha_{1}+\lambda_{2} \alpha_{1}=0, \quad \lambda_{2} \alpha_{2}=\lambda_{2} \alpha_{1} \beta_{1} \alpha_{2}=\lambda_{1} \alpha_{1} \beta_{1} \alpha_{2}=\lambda_{1} \alpha_{2}, \quad \xi=\lambda_{1}\left(\gamma-\alpha_{1}+\alpha_{1}-\alpha_{2}\right)$ $=\lambda_{1}\left(\gamma-\alpha_{2}\right) \in R\left(\gamma-\alpha_{2}\right)$. If $\xi \in f\left(z_{1}\right)$, then $\xi=\lambda_{1}\left(\delta-\alpha_{1}\right)+\lambda_{2} \epsilon_{2}=\mu_{1} \epsilon_{0}+\mu_{2}\left(\alpha_{1}-\alpha_{2}\right)$ for some $\lambda_{1}, \lambda_{2}, \mu_{1}, \mu_{2} \in R$. Hence $\xi \epsilon_{1}=-\lambda_{1} \alpha_{1}=\mu_{2} \alpha_{1}$ and $\xi \epsilon_{2}=\lambda_{2} \epsilon_{2}=-\mu_{2} \alpha_{2}$, so 
that $\lambda_{2} \epsilon_{2}=-\mu_{2} \alpha_{2}=-\mu_{2} \alpha_{1} \beta_{1} \alpha_{2}=\lambda_{1} \alpha_{1} \beta_{1} \alpha_{2}=\lambda_{1} \alpha_{2}$ and consequently $\xi=\lambda_{1}\left(\delta-\alpha_{1}+\alpha_{2}\right) \in R\left(\delta-\alpha_{1}+\alpha_{2}\right)$. It follows that if $\xi \in f\left(z_{2}\right)$, then $\xi=\lambda_{1}\left(\gamma-\alpha_{2}\right)$ $+\lambda_{2}\left(\delta-\alpha_{1}+\alpha_{2}\right)=\mu_{1} \epsilon_{0}+\mu_{2} \epsilon_{1}$ for some $\lambda_{1}, \lambda_{2}, \mu_{1}, \mu_{2} \in R$. Therefore $\xi \epsilon_{2}=-\lambda_{1} \alpha_{2}$ $+\lambda_{2} \alpha_{2}=0, \lambda_{2} \epsilon_{0}=\lambda_{2} \alpha_{2} \beta_{2}=\lambda_{1} \alpha_{2} \beta_{2}=\lambda_{1} \epsilon_{0}, \quad \xi=\lambda_{1}\left(\gamma-\alpha_{2}+\delta-\alpha_{1}+\alpha_{2}\right)=\lambda_{1}\left(\gamma+\delta-\alpha_{1}\right)$ $\in R\left(\gamma+\delta-\alpha_{1}\right)$. Thus we see that $f\left(z_{2}\right) \subseteq R\left(\gamma+\delta-\alpha_{1}\right)$, but on the other hand it is easy to check that $\gamma-\alpha_{2} \in f\left(z_{0}\right)$ and $\delta-\alpha_{1}+\alpha_{2} \in f\left(z_{1}\right)$, and hence $\gamma+\delta-\alpha_{1}$ $\in f\left(z_{2}\right)$. We therefore conclude that

$$
f\left(z_{2}\right)=R\left(\gamma+\delta-\alpha_{1}\right)=f g(\gamma+\delta)=f(v \oplus w),
$$

which shows that $v \oplus w=z_{2}$ and therefore proves (1).

If $\xi \in f\left(z_{3}\right)$, then $\xi=\lambda_{1}\left(\gamma-\alpha_{1}\right)+\lambda_{2}\left(\epsilon_{0}-\alpha_{2}\right)=\mu_{1} \epsilon_{1}+\mu_{2} \epsilon_{2}$ for some $\lambda_{1}, \lambda_{2}, \mu_{1}, \mu_{2}$ $\in R$. Therefore $\xi \epsilon_{0}=\lambda_{1} \gamma+\lambda_{2} \epsilon_{0}=0$, so that $\xi=\lambda_{1}\left(\gamma-\alpha_{1}\right)-\lambda_{1} \gamma\left(\epsilon_{0}-\alpha_{2}\right)$ $=\lambda_{1}\left(\gamma \alpha_{2}-\alpha_{1}\right) \in R\left(\gamma \alpha_{2}-\alpha_{1}\right)$. Since $z_{4}$ is obtained from $z_{0}$ by replacing $v$ by $w$, we see that $f\left(z_{4}\right) \subseteq R\left(\delta-\alpha_{2}\right)$. Consequently, if $\xi \in f\left(z_{6}\right)$, then $\xi=\lambda_{1}\left(\gamma \alpha_{2}-\alpha_{1}\right)$ $+\lambda_{2}\left(\delta-\alpha_{2}\right)=\mu_{1} \epsilon_{0}+\mu_{2} \epsilon_{1}$ for some $\lambda_{1}, \lambda_{2}, \mu_{1}, \mu_{2} \in R$. Therefore $\xi \beta_{2}=\lambda_{1} \gamma \alpha_{2} \beta_{2}$ $-\lambda_{2} \alpha_{2} \beta_{2}=0, \lambda_{1} \gamma=\lambda_{2} \epsilon_{0}$, so that $\xi=\lambda_{1}\left(\gamma \delta-\alpha_{1}\right) \in R\left(\gamma \delta-\alpha_{1}\right)$. On the other hand we easily see that $\gamma \alpha_{2}-\alpha_{1} \in f\left(z_{3}\right)$ and $\delta-\alpha_{2} \in f\left(z_{3}\right)$, whence it follows that $\gamma \delta-\alpha_{1} \in f\left(z_{5}\right)$, and we conclude that

$$
f\left(z_{\mathrm{b}}\right)=R\left(\gamma \delta-\alpha_{1}\right)=f g(\gamma \delta)=f(v \odot w),
$$

and therefore $z_{5}=v \odot w$. This proves (2).

As regard $g\left(\eta_{i}\right)$, it is easy to verify that

$$
g\left(\eta_{i}\right)=\left(x_{i}+b_{1}\right) c_{1}+\left(y_{i}+c_{1}\right) b_{1} \text {. }
$$

In fact,

$$
\begin{aligned}
f\left(\left(x_{i}+b_{1}\right) c_{1}\right) & =\left(R \eta_{i}+R \epsilon_{1}\right) \cap R\left(\epsilon_{0}-\alpha_{1}\right)=R \eta_{i}\left(\eta_{i}-\alpha_{1}\right), \\
f\left(\left(y_{i}+c_{1}\right) b_{1}\right) & =\left(R\left(\epsilon_{0}-\eta_{i}\right)+R\left(\epsilon_{0}-\alpha_{1}\right)\right) \cap R \epsilon_{1}=R\left(\eta_{i} \alpha_{1}-\alpha_{1}\right) \\
& =R\left(\epsilon_{0}-\eta_{i}\right)\left(\eta_{i}-\alpha_{1}\right) .
\end{aligned}
$$

Therefore $f$ maps the right hand side of (3) into

$$
R \eta_{i}\left(\eta_{i}-\alpha_{1}\right)+R\left(\epsilon_{i}-\eta_{i}\right)\left(\eta_{i}-\alpha_{1}\right)=R\left(\eta_{i}-\alpha_{1}\right)=f g\left(\eta_{i}\right) .
$$

Considering now $R^{\prime}$ in place of $R$, we obtain a one-to-one mapping $g^{\prime}$ of $\epsilon_{0}^{\prime} R \epsilon_{0}^{\prime}$ onto $T$ such that $g f\left(\gamma^{\prime}\right)=R^{\prime}\left(\gamma^{\prime}-\alpha_{1}^{\prime}\right)$ for all $\gamma^{\prime} \in \epsilon_{0}^{\prime} R^{\prime} \epsilon_{0}^{\prime}$, and this gives rise to operations with respect to which $T$ is a regular ring and an isomorphic image of $\epsilon_{0}^{\prime} R^{\prime} \epsilon_{0}^{\prime}$. However, since $\oplus$ and $\odot$ can be expressed in terms of the lattice $B$ and do not depend on the ring $R$, we see that the new operations must actually coincide with the old ones. Therefore there exists an isomorphism $h$ of $\epsilon_{0} R \epsilon_{0}$ onto $\epsilon_{0}^{\prime} R \epsilon_{0}^{\prime}$ such that $g^{\prime} h=g$. Finally, in view of (3) and the corresponding equation which must hold for $g^{\prime}\left(\eta_{i}^{\prime}\right)$, we see that $h\left(\eta_{i}\right)=\eta_{i}^{\prime}$.

The proof of the theorem is now complete.

10. Examples of simple complemented modular lattices. Von Neumann (cf. [10]) constructed two kinds of simple complemented modular lattices. The lattices of the first kind he obtained as limits of finite dimensional non- 
degenerate projective geometries, and observing that these lattices possess a positive valuation (dimension function), he was then able to complete them with respect to the resulting metric, thereby obtaining the lattices of the second kind, which are of course continuous geometries. Both types of lattices possess a global $n$-frame with $n \geqq 4$, and von Neumann's coordinatization theorem does therefore apply to them. By a modification of von Neumann's first construction we shall exhibit a simple complemented modular lattice, or actually a whole class of such lattices, for which this is not true. Our lattices do possess a positive valuation, but when the valuation is normalized by assigning the value 1 to the unit element, then there are no elements taking the values $1 / n, n=2,3,4, \cdots$. As far as we know it is an open question whether there exists a simple complemented modular lattice which does not possess a positive valuation.

TheOREM 10.1. There exists an infinite dimensional simple complemented modular lattice which does not possess a global $n$-frame for any integer $n>1$.

Proof. Choosing an infinite sequence of positive integers $k_{0}, k_{1}, k_{2}, \cdots$, let $m_{0}=1, m_{1}=k_{0}$ and $m_{i+1}=m_{i} k_{i}+m_{i-1}$ for $i=1,2,3, \cdots$. Let $F$ be any field (division ring) and let $B_{i}$ be a lattice which is isomorphic to the lattice of all subspaces of an $m_{i}-1$ dimensional nondegenerate projective space whose coordinate field is $F$. The dimension of $B_{i}$ is therefore $m_{i}$.

The unit element $u_{i+1}$ of $B_{i+1}$ can be written as a sum

$$
u_{i+1}=\sum_{j<k_{i}} v_{i, j}+w_{i}
$$

where each of the elements $v_{i, j}$ is $m_{i}$ dimensional and $w_{i}$ is $m_{i-1}$ dimensional. Hence there exists an isomorphism $p_{i, j}$ of $B_{i}$ onto $B_{i+1} v_{i, j}$, and an isomorphism $q_{i}$ of $B_{i-1}$ onto $B_{i+1} w_{i}$. The sublattice $C_{i}$ of $B_{i+1}$ which is generated by the ideals $B_{i+1} v_{i, j}, j<k_{i}$, and $B_{i+1} w_{i}$ is the (inner) direct product of these ideals, and we can therefore map the (outer) direct product $B_{i} \times B_{i-1}$ isomorphically into $C_{i}$ by letting

$$
f_{i}(\langle x, y\rangle)=\sum_{j<k_{i}} p_{i, j}(x)+q_{i}(y)
$$

for all $x \in B_{i}$ and $y \in B_{i-1}$. Finally we map $B_{i} \times B_{i-1}$ isomorphically into $B_{i+1} \times B_{i}$ by letting

$$
g_{i}(\langle x, y\rangle)=\left\langle f_{i}(\langle x, y\rangle), x\right\rangle .
$$

We now define our lattice $B$ to be the limit of the lattices $\dot{B}_{i} \times B_{i-1}$ with respect to the isomorphisms $g_{i}$. Thus there exist isomorphisms $h_{i}$ of $B_{i} \times B_{i-1}$ into $B$ for $i=1,2,3, \cdots$, such that $h_{i+1} g_{i}=h_{i}$, and such that every element of $B$ is in the image of $B_{i} \times B_{i-1}$ for sufficiently large $i$. Since

$$
g_{i}\left(\left\langle u_{i}, u_{i-1}\right\rangle\right)=\left\langle u_{i+1}, u_{i}\right\rangle,
$$

we see that $B$ has a unit element, and since the lattices $B_{i} \times B_{i-1}$ are complemented and modular it follows that $B$ is also complemented and modular. 
If $E$ is a congruence relation over $B$, then we can define a congruence relation $E_{i}$ over $B_{i} \times B_{i-1}$ by the condition that $\langle x, y\rangle E_{i}\left\langle x^{\prime}, y^{\prime}\right\rangle$ if and only if $h_{i}(\langle x, y\rangle) E h_{i}\left(\left\langle x^{\prime}, y^{\prime}\right\rangle\right)$. It follows that the conditions $\langle x, y\rangle E_{i}\left\langle x^{\prime}, y^{\prime}\right\rangle$ and $g_{i}(\langle x, y\rangle) E_{i+1} g_{i}\left(\left\langle x^{\prime}, y^{\prime}\right\rangle\right)$ are equivalent. Now suppose $0 \neq z \in B$ and $z E 0$. Then for a sufficiently large positive integer $i$ we have $z=h_{i}\left(\left\langle x_{i}, x_{i-1}\right\rangle\right)$ where $x_{i} \in B_{i}$ and $x_{i-1} \in B_{i-1}$. Furthermore

$$
g_{i}\left(\left\langle x_{i}, x_{i-1}\right\rangle\right)=\left\langle x_{i+1}, x_{i}\right\rangle, \quad g_{i+1}\left(\left\langle x_{i+1}, x_{i}\right\rangle\right)=\left\langle x_{i+2}, x_{i+1}\right\rangle
$$

where $x_{i+1} \in B_{i+1}$ and $x_{i+2} \in B_{i+2}$. Since $x_{i}$ and $x_{i-1}$ cannot both be equal to 0 , we must have $x_{i+1} \neq 0$ and $x_{i+2} \neq 0$, and inasmuch as $\left\langle x_{i+2}, x_{i+1}\right\rangle E_{i+2}\langle 0,0\rangle$, it follows by the simplicity of $B_{i+2}$ and $B_{i+1}$ that $\left\langle u_{i+2}, u_{i+1}\right\rangle E_{i+2}\langle 0,0\rangle$. Consequently $u E 0$ where $u$ is the unit element of $B$. We have therefore shown that $B$ is simple.

Finally, suppose there exists a global $n$-frame $a$ of $B$. Then

$$
u=\sum_{j<n} a_{j}
$$

where the elements $a_{j}$ are pairwise perspective. For sufficiently large $i$ we can therefore find elements $x_{j} \in B_{i}$ and $y_{j} \in B_{i-1}$, for $j=0,1, \cdots, n-1$, such that $a_{j}=h_{i}\left(\left\langle x_{j}, y_{j}\right\rangle\right)$. Consequently

$$
\begin{gathered}
u_{i}=\sum_{j<n} x_{j} \text { and } u_{i-1}=\sum_{j<n} y_{j}, \\
x_{0} \sim x_{1} \sim \cdots \sim x_{n-1} \text { and } y_{0} \sim y_{1} \sim \cdots \sim y_{n-1} .
\end{gathered}
$$

It follows that both $m_{i}$ and $m_{i-1}$ are divisible by $n$. But from the recursive definition of the integers $m_{i}$ we see that $m_{i}$ and $m_{i-1}$ are relatively prime, and we therefore conclude that $n=1$.

\section{BIBLIOGRAPHY}

1. I. Amemiya, On the representation of complemented modular lattices, J. Math. Soc. Japan vol. 9 (1957) pp. 263-279.

2. G. Birkhoff, Lattice theory, rev. ed., New York, Amer. Math. Soc. Colloquium Publications, vol. $25,1948$.

3. O. Frink, Complemented modular lattices and projective spaces of infinite dimension, Trans. Amer. Math. Soc. vol. 60 (1946) pp. 452-467.

4. B. Jonsson, Extensions of von Neumann's coordinatization theorem, to appear in Lattice theory, Proceedings of Symposia in Pure Mathematics, vol. 2.

5. - Modular lattices and Desargues' theorem, Math. Scand. vol. 2 (1954) pp. 295-314.

6. - On the representation of lattices, Math. Scand. vol. 1 (1953) pp. 193-206.

7. - Representations of lattices. II, Bull. Amer. Math. Soc. vol. 60 (1954) p. 24.

8. F. Maeda, Kontinuierliche Geometrien, Berlin, Springer-Verlag, 1958.

9. J. von Neumann, Examples of continuous geometries, Proc. Nat. Acad. Sci. U.S.A. vol. 22 (1936) pp. 92-100.

10. - Lectures on continuous geometries. I, II, III, Princeton, 1936-1937.

UNIVERSITY OF MiNNESOTA, Minneapolis, Minnesota 